\title{
Exploring the relativistic regime with Newtonian hydrodynamics
}

\section{An effective gravitational potential for rapid rotation}

\author{
B. Müller ${ }^{1}$, H. Dimmelmeier ${ }^{1,2}$, and E. Müller ${ }^{1}$ \\ 1 Max-Planck-Institut für Astrophysik, Karl-Schwarzschild-Str. 1, 85741 Garching, Germany \\ e-mail: bjmuellr@mpa-garching.mpg.de \\ 2 Department of Physics, Aristotle University of Thessaloniki, 54124 Thessaloniki, Greece
}

Received 19 February 2008 / Accepted 12 June 2008

\begin{abstract}
We present the generalization of a recently introduced modified gravitational potential for self-gravitating fluids. The use of this potential allows for an accurate approximation of general relativistic effects in an otherwise Newtonian hydrodynamics code also in cases of rapid rotation. We test this approach in numerical simulations of astrophysical scenarios related to compact stars, like supernova core collapse with both a simplified and detailed microphysical description of matter, and rotating neutron stars in equilibrium. We assess the quality of the new potential, and demonstrate that it provides a significant improvement compared to previous formulations for such potentials. Newtonian simulations of compact objects employing such an effective relativistic potential predict inaccurate pulsation frequencies despite the excellent agreement of the collapse dynamics and structure of the compact objects with general relativistic results. We analyze and discuss the reason for this behavior.
\end{abstract}

Key words. gravitation - hydrodynamics - methods: numerical - relativity - stars: supernovae: general

\section{Introduction}

Rapid rotation plays an important role in numerical simulations of a number of astrophysical events connected to stellar core collapse and neutron star evolution, such as accretion-induced collapse (Dessart et al. 2006, 2007a), the collapsar scenario for long gamma-ray bursts (MacFadyen \& Woosley 1999; Dessart et al. 2007b), the phase-transition-induced collapse of a rotating neutron star to a quark star (Lin et al. 2006), or rotational instabilities like the low $T /|W|$ instability in a collapsing stellar core (Centrella et al. 2001), proto-neutron star (PNS; Shibata \& Sekiguchi 2005; Ott et al. 2005, 2007a), or cold neutron star (NS; Shibata et al. 2002, 2003; Ou et al. 2004; Ou \& Tohline 2006). In all of these cases, as compact objects are involved, general relativistic (GR) effects should be taken into account properly. Moreover, extremely high radial and/or rotational velocities may be encountered, and thus a relativistic description of self-gravity and of the flow dynamics may be required. However, as an elaborate treatment of the microphysics in the form of neutrino transport and a microphysical equation of state $(\mathrm{EoS})$ is crucial for some of the scenarios, and as multi-dimensional GR codes for numerical simulations may either be unavailable (as in the case of detailed Boltzmann neutrino transport) or computationally very expensive, most previous investigations relied on the Newtonian approximation for the strong gravitational field of the compact objects, and on Newtonian flow dynamics.

A straightforward and easy to implement way of overcoming the first, most serious deficiency has been suggested recently for supernova core collapse involving at most moderately rapid rotation (Rampp \& Janka 2002; Marek et al. 2006). The strategy presented in these studies is to treat relativistic gravity by using an effective relativistic gravitational potential, while retaining the equations of Newtonian hydrodynamics. This allows for a simple upgrade of existing Newtonian hydrodynamics codes to treat relativistic gravity in an approximate but quite accurate manner. Extensive tests of this approach have been presented for simulations of supernova core collapse in spherical symmetry including Boltzmann neutrino transport and a microphysical EoS (Liebendörfer et al. 2005; Marek et al. 2006). The results of these tests show an excellent agreement with those obtained by corresponding fully relativistic $1 \mathrm{D}$ radiation hydrodynamic simulations. In addition, Marek et al. (2006) (Paper I hereafter) who studied rotational core collapse with a simplified EoS find good agreement between the results obtained with an axisymmetric Newtonian hydrodynamics code extended by an effective relativistic gravitational potential and those obtained with a GR code for cores with slow and moderate rotation. However, for rapidly rotating cores the effective relativistic potentials considered by Marek et al. (2006) cannot reproduce the dynamics of the collapse correctly, and e.g. underestimate the maximum density at bounce by more than $30 \%$. In particular, their potential A yields excellent results in the spherical limit, but clearly fails for rapid rotation, while potential $\mathrm{R}$ performs slightly better in the latter case, but persistently overestimates the maximum density during the evolution for slow or no rotation.

The results of Paper I suggest that the concept of an effective relativistic gravitational potential can in principle be used to model compact stars with rapid rotation. However, additional corrections to account for stronger centrifugal forces are necessary. To this end, we introduce here a new effective relativistic potential which contains correction terms for the case of rapid rotation. We assess the applicability of the new potential to the scenarios listed above, by presenting comparisons with both GR simulations and Newtonian simulations using two of the effective relativistic potentials described in Paper I for a range of core collapse and NS test models. We explore the new effective 
relativistic potential in the context of stellar core collapse with non-negligible rotation using either a simplified EoS or a microphysical equation in combination with an approximate deleptonization scheme. Furthermore, in order to test whether the new effective relativistic potential is also appropriate for compact equilibrium configurations, we consider rotating equilibrium polytropes which serve as models for a cold NS. We investigate pulsations of these models and briefly discuss the applicability of effective relativistic potentials to NS asteroseismology.

All of the above mentioned astrophysical events are interesting sources of gravitational radiation. Therefore, the extraction of the gravitational wave $(\mathrm{GW})$ signal from numerical simulations (possibly using effective relativistic potentials) and the prediction of wave templates for current and future GW detectors of interferometric and resonant type is an important issue. To this end, we discuss the results of Obergaulinger et al. (2006), who used one of the effective relativistic potentials from Paper I and report an overestimation of the GW amplitudes of up to $50 \%$ compared to simulations in GR even for slow rotators. We resolve this discrepancy by introducing a formulation of the Newtonian quadrupole formula that is better suited for both Newtonian simulations with an effective relativistic potential and fully relativistic simulations. $G=1$.

Throughout the article we use geometrized units with $c=$

\section{Effective relativistic potential}

In previous work (Rampp \& Janka 2002, and Paper I) it was proposed to approximate the effects of GR in a Newtonian hydrodynamics code by solely substituting the Newtonian potential by an effective relativistic gravitational potential $\Phi_{\text {eff }}$ which mimics the deeper potential well of relativistic gravity. We first recapitulate some properties of the Newtonian potential in Sect. 2.1, of the effective relativistic (TOV) potential introduced by Rampp \& Janka (2002) in Sect. 2.2, and of its most accurate improvement for slow and moderate rotation from Paper I in Sect. 2.3. Then we present in Sect. 2.4 the new effective relativistic potential including rotational corrections for rapidly rotating configurations. Finally, in Sect. 2.5 we summarize the treatment of GR in the conformally flat approximation, which is applied both in some previous studies and also in the present investigation of effective relativistic potentials for supernova core collapse for the purpose of comparison.

\subsection{Newtonian gravitational potential for a self-gravitating fluid}

In a genuine Newtonian simulation, which we refer to as Case $N$ in the following (see also Paper I), the Newtonian hydrodynamic equations are solved in conjunction with the (multi-dimensional) Newtonian gravitational potential

$\Phi_{\mathrm{N}}=-\int_{V} \mathrm{~d} r^{\prime} \mathrm{d} \theta^{\prime} \mathrm{d} \varphi^{\prime} r^{\prime 2} \sin \theta^{\prime} \frac{\rho}{\left|\boldsymbol{r}-\boldsymbol{r}^{\prime}\right|}$,

where $\rho$ is the (rest-mass) density of the self-gravitating fluid. This potential is the solution of the Poisson equation

$\Delta \Phi_{\mathrm{N}}=4 \pi \rho$.

For later use we also define the spherical part of the Newtonian potential by

$\bar{\Phi}_{\mathrm{N}}(r)=-\int_{\mathrm{r}}^{\infty} \frac{\mathrm{d} r^{\prime}}{r^{\prime 2}} \bar{m}_{\mathrm{N}}$ and the spherical Newtonian mass as

$\bar{m}_{\mathrm{N}}(r)=4 \pi \int_{0}^{r} \mathrm{~d} r^{\prime} r^{\prime 2}\langle\rho\rangle$

where any hydrodynamic quantity enclosed by brackets, $\langle\ldots\rangle$, is the angular average of its multi-dimensional counterpart.

\subsection{TOV potential for a self-gravitating fluid}

For devising an effective relativistic potential for a selfgravitating perfect fluid we require that for a spherically symmetric configuration it should reproduce the TolmanOppenheimer-Volkoff (TOV) solution of hydrostatic equilibrium in GR. Then a first approximation to include relativistic effects in a Newtonian code is to replace the spherical part $\bar{\Phi}(r)$ of the gravitational potential $\Phi(r, \theta, \phi)$ by the spherical TOV potential $\bar{\Phi}_{\mathrm{TOV}}$ (Rampp \& Janka 2002):

$\Phi_{\mathrm{R}}=\Phi_{\mathrm{N}}-\bar{\Phi}_{\mathrm{N}}+\bar{\Phi}_{\mathrm{TOV}}$

As in Paper I we refer to this effective relativistic potential as Case $R$ (or "reference" case). The spherical TOV potential is defined as

$\bar{\Phi}_{\mathrm{TOV}}(r)=-\int_{\mathrm{r}}^{\infty} \frac{\mathrm{d} r^{\prime}}{r^{\prime 2}}\left(\bar{m}_{\mathrm{TOV}}+4 \pi r^{\prime 3}\langle P\rangle\right) \frac{\langle h\rangle}{\bar{\Gamma}_{\mathrm{TOV}}^{2}}$,

where $h=1+\epsilon+P / \rho$ is the relativistic specific enthalpy, $\epsilon$ is the specific internal energy, and $P$ is the fluid pressure. The spherical TOV mass is given by

$\bar{m}_{\mathrm{TOV}}(r)=4 \pi \int_{0}^{r} \mathrm{~d} r^{\prime} r^{\prime 2}\langle\rho\rangle(1+\langle\epsilon\rangle)$,

and

$\bar{\Gamma}_{\mathrm{TOV}}(r)=\sqrt{1+\left\langle v_{\mathrm{r}}\right\rangle^{2}-\frac{2 \bar{m}_{\mathrm{TOV}}}{r}}$

with $v_{\mathrm{r}}$ being the Eulerian radial fluid velocity.

\subsection{Improved potential for a self-gravitating fluid without rotational corrections}

Marek et al. (2006) found in their study that the effective relativistic potential $\Phi_{R}$ overestimates the GR effects due to the straightforward combination of relativistic gravity and Newtonian dynamics. Thus, they proposed another effective relativistic potential

$\Phi_{\mathrm{A}} \equiv \Phi_{\mathrm{N}}-\bar{\Phi}_{\mathrm{N}}+\bar{\Phi}_{\mathrm{TOV}, \mathrm{A}}$,

labeled as Case A, which involves the modified spherical TOV potential

$\bar{\Phi}_{\mathrm{TOV}, \mathrm{A}}(r)=-\int_{\mathrm{r}}^{\infty} \frac{\mathrm{d} r^{\prime}}{r^{\prime 2}}\left(\bar{m}_{\mathrm{TOV}, \mathrm{A}}+4 \pi r^{\prime 3}\langle P\rangle\right) \frac{\langle h\rangle}{\bar{\Gamma}_{\mathrm{TOV}, \mathrm{A}}^{2}}$.

The latter differs from $\bar{\Phi}_{\mathrm{TOV}}(r)$ in Eq. (6) by the modified spherical TOV mass

$\bar{m}_{\mathrm{TOV}, \mathrm{A}}(r)=4 \pi \int_{0}^{r} \mathrm{~d} r^{\prime} r^{\prime 2}\langle\rho\rangle(1+\langle\epsilon\rangle) \bar{\Gamma}_{\mathrm{TOV}, \mathrm{A}}$, 
which compared to $\bar{m}_{\mathrm{TOV}}(r)$ in Eq. (7) contains an additional factor

$\bar{\Gamma}_{\mathrm{TOV}, \mathrm{A}}(r)=\sqrt{1+\left\langle v_{\mathrm{r}}\right\rangle^{2}-\frac{2 \bar{m}_{\mathrm{TOV}, \mathrm{A}}}{r}}$.

Note that this factor also appears explicitly in the definition of the modified spherical TOV potential $\bar{\Phi}_{\mathrm{TOV}, \mathrm{A}}(r)$ in Eq. (10).

Since $\bar{\Gamma}_{\text {TOV,A }}<1$ the modified spherical TOV mass $\bar{m}_{\mathrm{TOV}, \mathrm{A}}$ is smaller than $\bar{m}_{\text {TOV }}$. The fact that the right hand side of Eq. (11), which defines the modified spherical TOV mass $\bar{m}_{\mathrm{TOV}, \mathrm{A}}$, depends itself on $\bar{m}_{\mathrm{TOV}, \mathrm{A}}$, because of the factor $\bar{\Gamma}_{\mathrm{TOV}, \mathrm{A}}$, causes no problem, as $\bar{m}_{\mathrm{TOV}, \mathrm{A}}$ can be computed in a straightforward way by means of an ODE integration.

As demonstrated in Paper I, for simulations of rotational stellar core collapse the usage of the effective relativistic potential $\Phi_{\mathrm{A}}$ results in collapse dynamics and a PNS structure that are very close to the outcome in GR, at least in the regime of slow and moderately fast rotation.

\subsection{Effective relativistic potential with rotational corrections}

Following the concept of effective relativistic potentials discussed in Paper I, which are both easy to calculate and to implement in an existing Newtonian code, we require two criteria to be fulfilled for an effective relativistic potential $\Phi_{\text {Arot }}$ including corrections due to rotation (referred to as Case Arot in the following). Firstly, any modification should only involve the changes of the gravitational potential, thereby guaranteeing that the structure of the term $-\rho \nabla \Phi_{\mathrm{A}}$ in the momentum equation remains unchanged and is still integrable, and also that the Newtonian hydrodynamic equations remain unaltered. Secondly, in order to retain the proven accuracy of the effective relativistic potential $\Phi_{\mathrm{A}}$, the new potential $\Phi_{\text {Arot }}$ should reduce to that potential in the spherical limit.

For Cases $\mathrm{R}$ and $\mathrm{A}$ the effective relativistic potentials are computed by replacing only the monopole $\bar{\Phi}_{\mathrm{N}}$ of the Newtonian gravitational field $\Phi_{\mathrm{N}}$ with $\bar{\Phi}_{\mathrm{TOV}}$ or $\bar{\Phi}_{\mathrm{TOV}, \mathrm{A}}$, respectively. The obvious restriction of this approach is that any non-spherical (and in particular rotational) effects are accounted for exclusively in the higher multipoles of the Newtonian potential $\Phi_{N}$, limiting the GR corrections to the monopole. In contrast, Case Arot also involves GR corrections to the multipoles by modifying the source term of the Poisson Eq. (2).

Hence, we introduce a modified Newtonian potential $\Phi_{\mathrm{N} \text {,Arot }}$, whose spherical part $\bar{\Phi}_{\mathrm{N} \text {,Arot }}$ is (as in the other cases) substituted by the corresponding modified spherical TOV potential $\bar{\Phi}_{\text {TOV,Arot }}$ :

$\Phi_{\text {Arot }} \equiv \Phi_{\mathrm{N}, \text { Arot }}-\bar{\Phi}_{\mathrm{N}, \text { Arot }}+\bar{\Phi}_{\mathrm{TOV}, \text { Arot }}$.

The modified Newtonian potential $\Phi_{\mathrm{N}, \text { Arot }}$ is calculated by applying several changes to the source term in the Poisson Eq. (2). As a first step, we replace the density $\rho$ by $\rho W_{\Omega}$, where the angular Lorentz factor $W_{\Omega}=\left(1-v_{\Omega}^{2}\right)^{-1 / 2}$ with $v_{\Omega}^{2}=v_{\theta}^{2}+v_{\varphi}^{2}$ is restricted to the meridional and rotational velocity components $v_{\theta}$ and $v_{\varphi}$. With this choice, in the case of a purely angular velocity the density equals the conserved density in the GR continuity equation in the ADM $3+1$ split (see e.g. Banyuls et al. 1997). Disregarding the radial velocity component $v_{\mathrm{r}}$ in the Lorentz factor is motivated by the Birkhoff theorem, according to which neither the gravitational mass nor the vacuum field of a spherical star change if the matter is subject to radial motion only.
A comparison of the spherical Newtonian potential $\bar{\Phi}_{\mathrm{N}}$ with the (unmodified) spherical TOV potential $\bar{\Phi}_{\text {TOV }}$ suggests additional GR corrections for the source term of $\Phi_{\mathrm{N}, \text { Arot }}$. Neglecting the pressure term in Eq. (6) we get

$r^{2} \frac{\partial \bar{\Phi}_{\mathrm{TOV}}}{\partial r}=\bar{m}_{\mathrm{TOV}} \frac{\langle h\rangle}{\bar{\Gamma}_{\mathrm{TOV}}^{2}}$

and from Eq. (3)

$r^{2} \frac{\partial \bar{\Phi}_{\mathrm{N}}}{\partial r}=\bar{m}_{\mathrm{N}}$

By identifying $\bar{m}_{\text {TOV }}$ as a relativistic generalization of $\bar{m}_{\mathrm{N}}$, we obtain an additional correction factor $h / \Gamma_{\text {TOV,Arot }}^{2}$, which approximately takes into account the influence of the relativistic enthalpy $h$ and the self-interaction of the gravitational field in GR through $\Gamma_{\text {TOV,Arot }}$. The factor $h$ can also be inferred by comparing the gravity terms $\nabla \Phi_{\mathrm{N}}$ and $h \nabla v$ in the equations of rotating equilibrium configurations in Newtonian and GR gravity, respectively, where $v$ is a component of the spacetime metric which corresponds to the gravitational potential $\Phi_{N}$ in Newtonian gravity.

Thus, for calculating $\Phi_{\mathrm{N}, \text { Arot }}$ we finally arrive at

$\Delta \Phi_{\mathrm{N}, \text { Arot }}=4 \pi \rho_{\text {Arot }}$,

with the following expression for the source term:

$\rho_{\text {Arot }}=\frac{\rho h W_{\Omega}}{\Gamma_{\mathrm{TOV}, \mathrm{Arot}}^{2}}$

Accordingly, the effective relativistic potential $\Phi_{\mathrm{N}, \text { Arot }}$ can then be calculated in integral form as

$\Phi_{\mathrm{N}, \mathrm{Arot}}=-\int_{V} \mathrm{~d} r^{\prime} \mathrm{d} \theta^{\prime} \mathrm{d} \varphi^{\prime} r^{\prime 2} \sin \theta^{\prime} \frac{\rho_{\text {Arot }}}{\left|\boldsymbol{r}-\boldsymbol{r}^{\prime}\right|}$

and its spherical part as

$\bar{\Phi}_{\mathrm{N}, \text { Arot }}(r)=-\int_{0}^{\infty} \frac{\mathrm{d} r^{\prime}}{r^{\prime 2}} \bar{m}_{\mathrm{N}, \text { Arot }}$.

Here the modified spherical Newtonian mass is defined as

$\bar{m}_{\mathrm{N}, \mathrm{Arot}}(r)=4 \pi \int_{0}^{r} \mathrm{~d} r^{\prime} r^{\prime 2} \bar{\rho}_{\text {Arot }}$,

with the modified spherical density $\bar{\rho}_{\text {Arot }}$ given by

$\bar{\rho}_{\text {Arot }}=\frac{\langle\rho\rangle\langle h\rangle\left\langle W_{\Omega}\right\rangle}{\bar{\Gamma}_{\mathrm{TOV}, \text { Arot }}^{2}}$.

Note that $\bar{\rho}_{\text {Arot }}$ is not computed as the angular average of $\rho_{\text {Arot }}$ itself but rather of its constituents, because it is a quantity derived from other hydrodynamic quantities. The angular average of the Lorentz factor is given by $\left\langle W_{\Omega}\right\rangle=\left\langle\left(1-v_{\Omega}^{2}\right)^{-1 / 2}\right\rangle$.

For the modified spherical TOV potential of Case Arot we use an expression that is analogous to Eqs. (6) and (10):

$$
\begin{aligned}
\bar{\Phi}_{\mathrm{TOV}, \mathrm{Arot}}(r)= & -\int_{\mathrm{r}_{\mathrm{r}}}^{\infty} \frac{\mathrm{d} r^{\prime}}{r^{\prime 2}}\left(\bar{m}_{\mathrm{TOV}, \mathrm{Arot}}+4 \pi r^{\prime 3}\langle P\rangle\right) \\
& \times \frac{\langle h\rangle}{\bar{\Gamma}_{\mathrm{TOV}, \mathrm{Arot}}^{2}}\left\langle\left(1-v_{\Omega}^{2}\right)^{-1}\right\rangle_{\rho} .
\end{aligned}
$$

This potential also contains several additions related to nonspherical effects. First of all, in the modified spherical TOV mass

$\bar{m}_{\text {Arot }}(r)=4 \pi \int_{0}^{r} \mathrm{~d} r^{\prime} r^{\prime 2}\left\langle\rho W_{\Omega}\right\rangle(1+\langle\epsilon\rangle) \bar{\Gamma}_{\text {Arot }}$, 
the angular averaged density $\langle\rho\rangle$ is substituted by $\left\langle\rho W_{\Omega}\right\rangle$ with the same rationale as in the source term of the Poisson Eq. (16), while the definition of

$\bar{\Gamma}_{\text {TOV,Arot }}(r)=\sqrt{1+\left\langle v_{\mathrm{r}}\right\rangle^{2}-\frac{2 \bar{m}_{\mathrm{TOV}, \text { Arot }}}{r}}$

follows those of Eqs. (8) and (12). The introduction of the centrifugal factor

$$
\left\langle\left(1-v_{\Omega}^{2}\right)^{-1}\right\rangle_{\rho}=\frac{\left\langle\rho W_{\Omega}^{3}\right\rangle}{\left\langle\rho W_{\Omega}\right\rangle}
$$

in the effective relativistic potential (22) is suggested by comparing the equations for stationary equilibria of rotating selfgravitating fluids in Newtonian and GR gravity. In essence, the centrifugal factor results in a larger gravitational acceleration for high rotational velocities. It mimics a relativistic effect that is, for instance, known from the motion of a test particle in a Schwarzschild background spacetime, where the effective relativistic potential (see e.g. Straumann 2004)

$V_{\mathrm{eff}}=\left(1-\frac{2 m}{r}\right)\left(1+\frac{l^{2}}{r^{2}}\right)$

for the motion of a test particle with specific angular momentum $l=r v_{\Omega}$ contains a factor $\left(1+l^{2} / r^{2}\right)$, which is identical to $(1-$ $\left.v_{\Omega}^{2}\right)^{-1}$ to order $O\left(v^{2}\right)$. The density averaging is preferable to a volume averaging to account for the rotational flattening in cases of rapid rotation.

As required the new effective relativistic potential $\Phi_{\text {Arot }}$ by construction reduces to $\Phi_{\mathrm{A}}$ in spherical symmetry. In this limit the multi-dimensional and spherical (modified) Newtonian potentials are identical, $\Phi_{\mathrm{N}}=\bar{\Phi}_{\mathrm{N}}$ and $\Phi_{\mathrm{N}, \text { Arot }}=\bar{\Phi}_{\mathrm{N}, \text { Arot }}$. Thus, they cancel each other in Eqs. (9) and (13), respectively, while the modified TOV potentials $(10,22)$ become identical as $\left\langle W_{\Omega}\right\rangle$ vanishes.

Note that different from Paper I, for reason of simplicity we omit all contributions due to neutrino effects in the effective relativistic potentials discussed here. However, it is straightforward to add the appropriate terms to any of the potentials (see Paper I).

\subsection{General relativistic formulation using the conformally flat approximation}

To be able to directly assess the quality of the effective relativistic potential approach in describing rapidly rotating compact objects, we have repeated all simulations using a GR hydrodynamics code. These results are referred to as Case GR. As in Paper I, we approximate the GR spacetime metric by the conformal flatness condition (CFC; see Isenberg 1978; Wilson et al. 1996), whose excellent quality in the context of rotational stellar core collapse and a rotating NS has been demonstrated extensively (see e.g. Shibata \& Sekiguchi 2004; Cerdá-Durán et al. 2005; Ott et al. 2007a, and references therein).

\section{Numerical methods and models}

In order to comprehensively test the new effective relativistic potential in situations where centrifugal forces play an important role, we apply it to simulations of the collapse of a rotating stellar core to a PNS and of a rapidly rotating NS in equilibrium. In Sect. 3.1 we summarize the properties of the code used for these simulations, while in Sect. 3.2 we describe the setup of the initial models and the EoS for each of the two scenarios.

\subsection{Code and grid setup}

All simulations are performed with the code CoCoNuT of Dimmelmeier et al. (2002a, 2005) assuming axial symmetry and symmetry with respect to the equatorial plane. This code optionally either uses a Newtonian (or alternatively an effective relativistic) gravitational potential and Newtonian hydrodynamics (Cases N, R, A, and Arot) or solves the GR equations of fluid dynamics and the GR field equations in the ADM $3+1$ split assuming CFC (Case GR). This procedure allows for a direct comparison of the different effective relativistic potentials with GR using one numerical code and an identical grid setup.

The CoCoNuT code employs a metric solver based on spectral methods as described in Dimmelmeier et al. (2005). The (Newtonian or GR) hydrodynamic equations are formulated in conservation form, and are solved by high-resolution shock-capturing schemes with state-of-the-art Riemann solvers and piecewise parabolic cell reconstruction procedures on an Eulerian grid in spherical polar coordinates $\{r, \theta\}$.

Rotating NS are modelled with an equidistant grid of $80 \times$ 30 zones. For the simulations of stellar core collapse the radial grid is logarithmically spaced to increase the effective resolution in the center where the PNS forms. In that case the grid consists of $200 \times 30$ zones in $\{r, \theta\}$ with a central radial resolution of $\Delta r_{\mathrm{c}}=500 \mathrm{~m}$ for models with a simplified EoS, and of $250 \times 45$ zones with $\Delta r_{\mathrm{c}}=250 \mathrm{~m}$ for the models computed with a microphysical EoS. In both cases, the computational domain extends from $r_{\min }=0$ to $r_{\max }=2000 \mathrm{~km}$. Reflecting boundary conditions are imposed at the origin; otherwise no specific measures are taken to deal with the singularity at $r=0$. A small part of the grid is occupied by an artificial low-density atmosphere extending beyond the surface of the NS or stellar core. For more details about the grid setup and the boundary conditions, we refer to e.g. Dimmelmeier et al. (2005), Dimmelmeier et al. (2006), or Ott et al. (2007b).

\subsection{Equations of state, and models for rotating neutron stars or stellar core collapse}

As initial data for the simulations of NS we choose rotating equilibrium configurations which obey a polytropic EoS,

$P=K \rho^{\gamma}$

with $K=1.4553 \times 10^{5}$ (in cgs units ${ }^{1}$ ) and $\gamma=2$. The models are computed with the self-consistent field method of Komatsu et al. (1989). Their rotation law for the specific angular momentum $j$ is given by

$j=A^{2}\left(\Omega_{\mathrm{c}}-\Omega\right)$,

where $A$ parameterizes the degree of differential rotation (more differential for smaller values of $A$ ), and $\Omega_{\mathrm{c}}$ is the value of the angular velocity $\Omega$ at the center. In the Newtonian limit, this rotation law reduces to

$\Omega=\frac{A^{2} \Omega_{\mathrm{c}}}{A^{2}+\varpi^{2}}$,

where $\varpi$ is the distance to the rotation axis.

In the simulations of rotational core collapse with a simplified EoS the initial rotating equilibrium models are computed with a polytropic EoS. They approximate an iron core supported by electron degeneracy pressure with a central density $\rho_{\mathrm{c}, \mathrm{i}}=10^{10} \mathrm{~g} \mathrm{~cm}^{-3}$, and the EoS parameters $K=4.897 \times 10^{14}$

1 This value is commonly used for polytropic NS models. 
Table 1. Nomenclature of the iron core collapse models AxByGz (simulated with the hybrid EoS) and s20AxBy (simulated with the microphysical EoS). The length parameter $A$ specifies the initial degree of differential rotation, $(T /|W|)_{i}$ is the initial rotation rate, and $\gamma_{1}$ is the stiffness of the subnuclear part of the hybrid EoS, respectively. We also give the angular velocity at the center for the initial configuration $\left(\Omega_{\mathrm{c}, \mathrm{i}}\right)$ and at bounce $\left(\Omega_{\mathrm{c}, \mathrm{b}}\right)$. For models marked with an asterisk, we give the maximum angular velocity $\Omega_{\text {max,b }}$ instead of $\Omega_{\mathrm{c}, \mathrm{b}}$, because the density in the central region is extremely low due to the toroidal density stratification, and $\Omega_{\mathrm{c}, \mathrm{b}}$ is therefore no good indicator for the rotational state of the entire core.

\begin{tabular}{lccccc}
\hline \hline Model & $A[1000 \mathrm{~km}]$ & $(T /|W|)_{\mathrm{i}}[\%]$ & $\Omega_{\mathrm{c}, \mathrm{i}}\left[\mathrm{s}^{-1}\right]$ & $\Omega_{\mathrm{c}, \mathrm{b}}\left[10^{3} \mathrm{~s}^{-1}\right]$ & $\gamma_{1}$ \\
\hline A1B1G1 & 50.0 & 0.25 & 2.3 & 2.9 & 1.325 \\
A1B2G1 & 50.0 & 0.5 & 3.1 & 3.6 & 1.325 \\
A1B3G1 & 50.0 & 0.9 & 4.1 & 6.2 & 1.325 \\
A1B3G2 & 50.0 & 0.9 & 4.1 & 4.8 & 1.320 \\
A1B3G3 & 50.0 & 0.9 & 4.1 & 2.8 & 1.310 \\
A2B4G1 & 1.0 & 1.8 & 8.0 & 1.8 & 1.325 \\
A3B1G1 & 0.5 & 0.25 & 4.6 & 3.9 & 1.325 \\
A3B2G4 & 0.5 & 0.5 & 6.3 & 6.6 & 1.300 \\
A3B4G2 & 0.5 & 1.8 & 12.4 & 2.3 & 1.320 \\
A4B1G1 & 0.1 & 0.25 & 31.1 & 21 & 1.325 \\
A4B1G2 & 0.1 & 0.25 & 31.1 & 21 & 1.320 \\
A4B2G2 & 0.1 & 0.5 & 43.6 & 22 & 1.320 \\
A4B2G3 & 0.1 & 0.5 & 43.6 & 7.5 & 1.310 \\
A4B5G4 & 0.1 & 4.0 & 139.4 & $2.4^{*}$ & 1.300 \\
A4B5G5 & 0.1 & 4.0 & 139.4 & $2.5^{*}$ & 1.280 \\
s20A1B1 & 50.0 & 0.25 & 1.0 & 1.0 & - \\
s20A2B4 & 1.0 & 0.5 & 9.6 & 3.3 & - \\
\hline
\end{tabular}

and $\gamma=4 / 3$, respectively. For the evolution of the cores a hybrid EoS is employed (Janka et al. 1993; Dimmelmeier et al. 2002a). It consists of a polytropic contribution describing the degenerate electron pressure and (at supranuclear densities) the pressure due to repulsive nuclear forces, and a thermal contribution which accounts for shock heating:

$P=P_{\mathrm{p}}+P_{\mathrm{th}}$,

where

$P_{\mathrm{p}}=K \rho^{\gamma}, \quad P_{\mathrm{th}}=\rho \epsilon_{\mathrm{th}}\left(\gamma_{\mathrm{th}}-1\right)$,

with $\gamma_{\text {th }}=1.5$. To trigger the collapse the adiabatic index is reduced from its initial value $4 / 3$ to $\gamma_{1}<4 / 3$. At nuclear density $\rho_{\text {nuc }}=2 \times 10^{14} \mathrm{~g} \mathrm{~cm}^{-3}$ the adiabatic index is raised abruptly to a value $\gamma_{2}=2.5$ to mimic the stiffening of the nuclear EoS, which results in a rebound of the core (the core bounce) and the formation of the PNS. The hybrid EoS provides for a smooth transition between these two density regimes (for more details, see Janka et al. 1993).

In our microphysically more detailed calculations of stellar core collapse we employ the tabulated non-zero temperature nuclear EoS by Shen et al. (1998) in the variant of Marek et al. (2005) which includes pressure contributions from baryons, leptons, and photons. Deleptonization by electron capture onto nuclei and free protons is implemented according to Liebendörfer (2005): during collapse the electron fraction $Y_{\mathrm{e}}$ is parameterized as a function of density based on neutrino radiation-hydrodynamic simulations in spherical symmetry (Marek et al. 2005) using the electron capture rates of (Langanke \& Martínez-Pinedo 2000). After core bounce, $Y_{\mathrm{e}}$ is only passively advected, and any further lepton loss is neglected. Again following Liebendörfer (2005), above the trapping density $\rho_{\text {trap }}=2.0 \times 10^{12} \mathrm{~g} \mathrm{~cm}^{-3}$ contributions due to neutrino radiation pressure are taken into account. As initial models we take the non-rotating $20 \mathrm{M}_{\odot}$ solar-metallicity progenitor $\mathrm{s} 20.0$ from Woosley et al. (2002) and impose the rotation law (28). Note that these initial models are not in equilibrium, because the progenitor model s20.0 already shows considerable negative radial velocities and the models are not relaxed with respect to the rotation added.

In Table 1 we summarize the parameters of all investigated stellar core collapse models. They are characterized by their initial degree of differential rotation $A$ and by their initial rotation parameter $(T /|W|)_{i}$, which is the ratio of rotational energy to (the absolute value of the) gravitational binding energy. The corresponding angular velocity $\Omega_{\mathrm{c}, \mathrm{i}}$ of the progenitor model is also shown, as well as the angular velocity $\Omega_{\mathrm{c}, \mathrm{b}}$ at bounce. For models evolved using the hybrid EoS, the stiffness $\gamma_{1}$ of the subnuclear EoS is also specified.

Our selection of models includes fast-rotating cores which end up as PNSs with spin periods in the milisecond or submilisecond range. Lower initial rotation rates - as predicted by recent stellar evolution models incorporating magnetic braking (Heger et al. 2005) - are required to match the spin periods of observed young pulars, which are typically larger than $10 \mathrm{~ms}$. However, since our goal is to test the effective potential approach for such rotation rates as required by the scenarios (collapsars, etc.) mentioned in the introduction, we also consider rapidly rotating models. While rare in nature, there are viable formation channels for such progenitors: even when magnetic torques are included, it is still possible to obtain fast rotating cores for massive Wolf-Rayet stars for sufficiently low mass loss rates (Woosley \& Heger 2006) (which may be realistic for lowmetallicity stars), although few supernovae are expected to originate from such progenitors because of the declining initial mass function. Binary evolution effects, i.e. accretion (Cantiello et al. 2007; Yoon \& Langer 2005) or mergers (Fryer \& Heger 2005), may also lead to rapidly spinning cores. Again, only a small fraction of core collapse events can be explained this way: for the particular case of accretion-induced collapse, the most optimistic value quoted by Dessart et al. (2007a) is only $1.5 \times 10^{-4}$ galactic events per year. 
Table 2. Maximum density $\rho_{\max , \mathrm{b}}$ at bounce and final maximum density $\rho_{\text {max,f }}$ after ring-down in units of $10^{14} \mathrm{~g} \mathrm{~cm}^{-3}$ for all investigated core collapse models in Case GR, or evolved with the effective relativistic potential $\Phi_{R}$, and $\Phi_{\text {Arot }}$, respectively. For each potential, the relative deviation from Case GR (in percent) is given in parentheses, and the value closest to Case GR is indicated by an asterisk. Note that some centrifugally bouncing models do not reach stable values for $\rho_{\max , \mathrm{f}}$ until very long after core bounce.

\begin{tabular}{lcccccccc}
\hline \hline & \multicolumn{3}{c}{$\rho_{\text {max,b }}$} & & \multicolumn{3}{c}{$\rho_{\text {max,f }}$} \\
Model & Case GR & Case R & Case A & Case Arot & Case GR & Case R & Case A & Case Arot \\
\hline A1B1G1 & 4.71 & $4.55(-3)^{*}$ & $4.32(-8)$ & $4.42(-6)$ & 3.30 & $3.53(+7)$ & $3.36(+2)^{*}$ & $3.40(+3)$ \\
A1B2G1 & 4.37 & $4.26(-3)^{*}$ & $3.98(-9)$ & $4.16(-5)$ & 3.16 & $3.32(+5)$ & $3.19(+1)^{*}$ & $3.25(+3)$ \\
A1B3G1 & 3.86 & $3.78(-2)$ & $3.56(-7)$ & $3.81(-1)^{*}$ & 2.84 & $3.00(+6)$ & $2.82(-1)^{*}$ & $2.97(+5)$ \\
A1B3G2 & 4.02 & $3.88(-3)$ & $3.70(-8)$ & $3.90(-3)^{*}$ & 2.78 & $2.88(+4)$ & $2.78( \pm 0)^{*}$ & $2.84(+2)$ \\
A1B3G3 & 4.19 & $4.04(-4)^{*}$ & $3.93(-6)$ & $4.01(-4)$ & 2.99 & $2.11(+4)$ & $3.03(+1)^{*}$ & $3.10(+4)$ \\
A2B4G1 & 0.64 & $0.44(-31)$ & $0.30(-53)$ & $0.58(-9)^{*}$ & - & - & - & - \\
A3B1G1 & 4.46 & $4.27(-4)^{*}$ & $4.05(-9)$ & $4.25(-5)$ & 3.16 & $3.33(+5)$ & $3.18(+1)^{*}$ & $3.26(+4)$ \\
A3B2G4 & 3.98 & $3.83(-4)$ & $3.76(-6)$ & $3.87(-3)^{*}$ & 2.54 & $2.57(+1)$ & $2.54( \pm 0)^{*}$ & $2.60(+2)$ \\
A3B4G2 & 0.59 & $0.41(-31)$ & $0.33(-44)$ & $0.58(-1)^{*}$ & - & - & - & - \\
A4B1G1 & 4.64 & $4.16(-7)$ & $3.94(-15)$ & $4.30(-10)^{*}$ & 2.98 & $2.96(-1)^{*}$ & $2.85(+4)$ & $3.09(+4)$ \\
A4B1G2 & 4.46 & $4.12(-8)$ & $3.88(-7)$ & $4.23(-5)^{*}$ & 2.84 & $2.83( \pm 0)^{*}$ & $2.75(-3)$ & $2.96(+4)$ \\
A4B2G2 & 4.31 & $3.59(-17)$ & $3.47(-20)$ & $4.08(-2)^{*}$ & 2.56 & $2.37(-7)$ & $2.30(-10)$ & $2.72(+6)^{*}$ \\
A4B2G3 & 4.00 & $4.46(+11)$ & $3.38(-16)$ & $3.91(-5)^{*}$ & 2.30 & $2.14(-7)$ & $1.55(-33)$ & $2.42(+5)^{*}$ \\
A4B5G4 & 0.47 & $0.36(-25)$ & $0.35(-27)$ & $0.51(+7)^{*}$ & - & - & - & - \\
A4B5G5 & 1.72 & $1.26(-27)$ & $1.26(-27)$ & $1.72( \pm 0)^{*}$ & - & - & - & - \\
s20A1B1 & 3.21 & $3.14(-2)^{*}$ & $3.06(-5)$ & $3.07(-4)$ & 2.69 & $2.78(+3)$ & $2.73(+1)^{*}$ & $2.74(+2)$ \\
s20A2B4 & 1.61 & $1.18(-27)$ & $0.89(-45)$ & $1.64(+2)^{*}$ & 0.94 & $0.75(-20)^{*}$ & $0.62(-34)$ & $1.18(+26)$ \\
\hline
\end{tabular}

\section{Results}

\subsection{Dynamics of rotational stellar core collapse}

Previous simulations, considering a large variety of rotation rates and profiles in the progenitor core but simplifying the complex microphysics and/or the influence of GR, found qualitatively and quantitatively different types of GW burst signals (see e.g. the work by Mönchmeyer et al. 1991; Zwerger \& Müller 1997; Dimmelmeier et al. 2002b). These can be classified depending on the collapse dynamics: Type I signals are emitted when the collapse of the homologously contracting inner core is not strongly influenced by rotation, but stopped by a pressuredominated bounce due to the stiffening of the EoS above nuclear matter density. This leads to the formation of the PNS with a maximum core density $\rho_{\max } \geq \rho_{\text {nuc }}$ after a few ring-down oscillations. Type II signals occur when centrifugal forces, which grow during contraction due to angular momentum conservation, are sufficiently strong to halt the collapse, resulting in consecutive (typically multiple) centrifugal bounces with intermediate coherent re-expansion of the inner core, seen as density drops by often more than an order of magnitude; thus here $\rho_{\max }<\rho_{\text {nuc }}$ after bounce. Type III signals result from a pressure-dominated bounce when the inner core has a very small mass at bounce due to a soft subnuclear EoS or very efficient electron capture.

Accordingly, we discuss the quality of the new effective relativistic potential by first applying it to rotating stellar cores which collapse to form a PNS, focusing on three different cases: a regular pressure-dominated bounce model with slow or moderate rotation, a centrifugal single bounce model with rapid and strongly differential rotation (characterized by a marked toroidal density stratification ${ }^{2}$ that develops in the core), and a multiple centrifugal bounce model. As the type III bounce models have very similar dynamics to type I models, we refrain from discussing them separately. The reader should refer to Table 2 throughout the discussion, as it presents key quantities (namely

\footnotetext{
2 In this paper, a configuration will be called "toroidal" if the maximum density is reached off-center.
}

the maximum density at bounce and the final maximum density) for our entire set of models, thus providing further illustrative examples for moderately rotating cores with weak (A1 models, s20A1B1) and strong (most A3 and A4 models) differential rotation, as well as multiple (A2B4G1, A3B4G2) and single (A4B5G5, A4B5G5 and s20A2B4) centrifugal bounce models.

\subsubsection{Slow and moderate rotation with pressure-dominated bounce}

For the regular collapse models with slow or moderate rotation we essentially confirm the findings of Paper I, as the effective relativistic potentials $\Phi_{\text {Arot }}$ and $\Phi_{\mathrm{A}}$ are identical in the spherical limit. For the models $\mathrm{A} 1 \mathrm{~B} 3 \mathrm{G} 3$ and $\mathrm{A} 4 \mathrm{~B} 1 \mathrm{G} 2$ presented in Paper I, as well as for all our other models with the simple hybrid EoS which undergo pressure-dominated bounce, $\Phi_{\text {Arot }}$ yields results that are in very good agreement with those of GR simulations. In particular, after bounce and ring-down $\rho_{\max }$ is reproduced to an accuracy of $7 \%$ or better (see Table 2 ). Note that for these nine models $(T /|W|)_{\mathrm{b}}<0.1$. The maximum density $\rho_{\max , \mathrm{b}}$ at bounce is usually a bit underestimated, but correct to within $10 \%$. Typically, Case A matches Case GR most closely in the post-bounce phase, followed by Case Arot (the new potential including rotation corrections) and Case $\mathrm{R}$ (the potential with pure TOV corrections), while Case Arot works best during bounce. This behaviour is illustrated for model A1B1G1 in Fig. 1. The agreement is even better for the microphysical model s20A1B1, whose initial rotation rate and profile is identical to that of model A1B1G1. Here the error in $\rho_{\max }$ (compared to the GR case) is smaller than $5 \%$ at bounce (where now Case R agrees best with Case GR) and 3\% after ring-down, as summarized in Table 2.

\subsubsection{Multiple centrifugal bounce}

The limits in applying the old effective relativistic potentials presented in Paper I (Cases A and R) become apparent for models which collapses slowly due to $\gamma_{1}$ being close to $4 / 3$ and are 


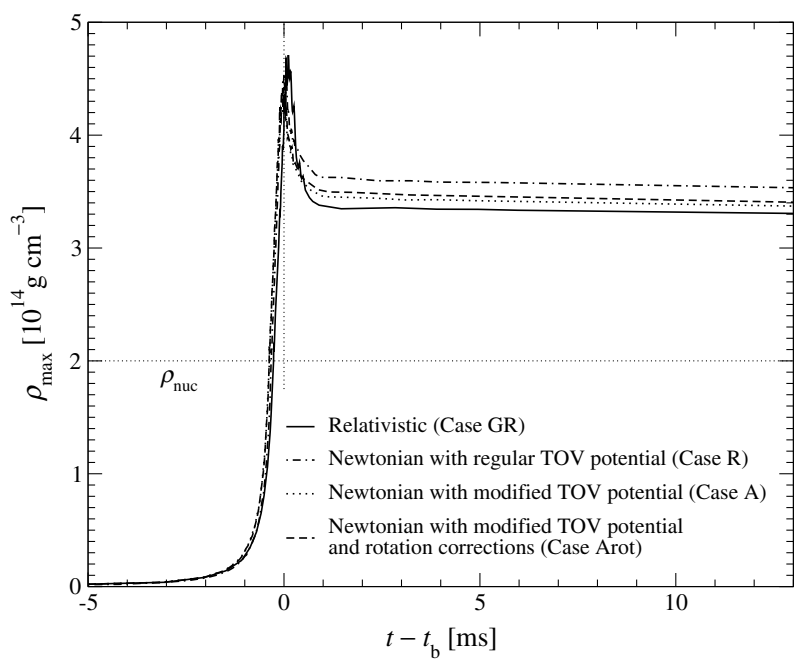

Fig. 1. Time evolution of the maximum density $\rho_{\max }$ for the slowly and almost uniformly rotating model A1B1G1 for Case GR (solid line), Case R (dashed-dotted line), Case A (dotted line), and Case Arot (dashed line), respectively. The results of all Newtonian simulations with effective relativistic potentials differ from the GR results by only a few percent. $t_{\mathrm{b}}$ is the time of core bounce.

strongly influenced by centrifugal forces due to a considerable amount of initial rotation. Such models undergo multiple centrifugal bounces where always $\rho_{\max }<\rho_{\text {nuc }}$, and do not settle down to a quasi-equilibrium state even after several bounces, and the resulting waveform of the emitted gravitational radiation is of type II. It should be emphasized that multiple bounces do no longer occur once deleptonization is taken into account, essentially because a choice of $\gamma_{1}$ very close to $4 / 3$ does not constitute an adequate approximation for the dynamics of the collapse. However, a rather high value of $\gamma_{1}$, such as to produce multiple bounces, still serves as an interesting test case which allows us to check the corrections due to rotation included for Case Arot in a regime where centrifugal forces dominate the evolution of the core around bounce. For this reason, we consider one model (A2B4G1) which provides a good example for the multiple bounce scenario.

In this model (and comparable models), $\rho_{\max , \mathrm{b}}$ at the first bounce is underestimated by more than $50 \%$ in Case $\mathrm{A}$ and by more than $30 \%$ in Case R (see Fig. 2, and also Fig. 13 in Paper I), while the use of the new potential $\Phi_{\text {Arot }}$ reduces this error to less than $10 \%$. This is an extraordinary result, as in a collapse situation with multiple centrifugal bounces the evolution of $\rho_{\max }$ sensitively depends on the balance between centrifugal forces and the pressure gradient for an EoS with a $\gamma_{1}$ close to $4 / 3$.

For rotational core collapse simulations with a simple hybrid EoS, the appropriate modeling of GR effects (via a consistent relativistic hydrodynamic formulation like in Case GR or by the use of the new effective relativistic potential $\Phi_{\text {Arot }}$ in an otherwise Newtonian framework) strongly reduces the range in parameter space where multiple centrifugal bounce models occur (Dimmelmeier et al. 2002b). Moreover, independent of the inclusion of relativistic gravity, only models with $\gamma_{1} \geq 1.31$ will exhibit such a bounce behavior, even in a purely Newtonian treatment. However, as will be explained in detail in the next Sect. 4.1.3, the occurence of models showing multiple centrifugal bounces results from the use of a simplified EoS, and vanishes if an appropriate microphysical matter model is applied.

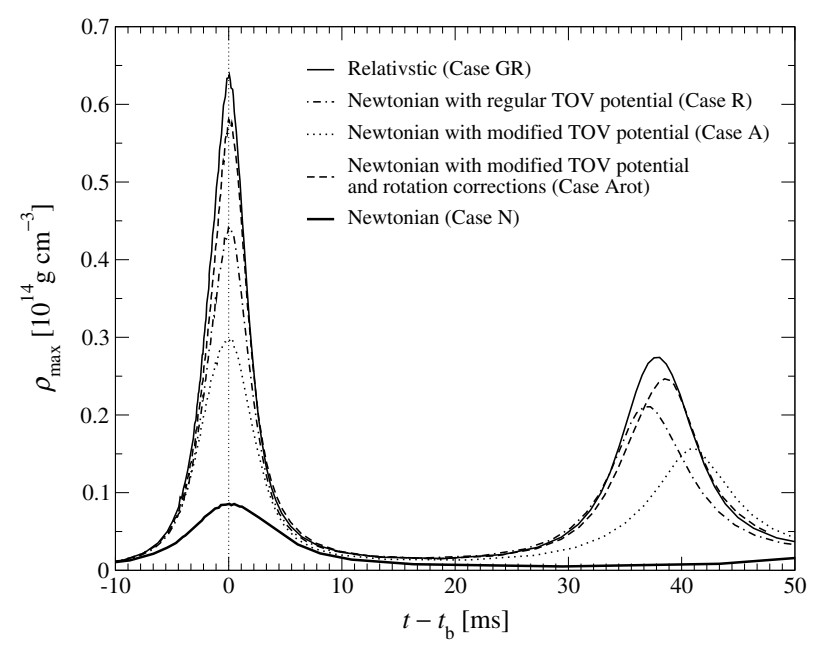

Fig. 2. Same as Fig. 1 but for the multiple centrifugal bounce model A2B4G1. The effective relativistic potential $\Phi_{\text {Arot }}$ reproduces the GR result to within $10 \%$ even though this model is strongly influenced by centrifugal forces. The additionally shown purely Newtonian simulation grossly underestimates $\rho_{\max }$.

\subsubsection{Rapid and strongly differential rotation with single centrifugal bounce}

If the microphysical EoS and the approximate deleptonization scheme described in Sect. 3.2 are employed, the collapse dynamics and the GW burst signature are exclusively of type I. This was first observed by Ott et al. (2007a), and then confirmed by Dimmelmeier et al. (2007) who considerably extended the number of investigated models and comprehensively explored a wide parameter space of initial rotation states. They found that the combination of deleptonization during core collapse and (to a lesser degree) the influence of GR lead to this emergence of a generic GW signal type. In most microphysical simulations, the core undergoes a regular pressure-dominated bounce, which results in a dynamical behavior that is well represented by the evolution of $\rho_{\max }$ shown in Fig. 1. Accordingly, the new effective relativistic potential $\Phi_{\text {Arot }}$ as well as potentials $\Phi_{\mathrm{A}}$ and $\Phi_{\mathrm{R}}$ again yield excellent results, as they also do for models based on a simple hybrid EoS (see model s20A1B1 in Table 2).

Only for models with very fast initial rotation (with an initial value ${ }^{3}$ of $T /|W| \gtrsim 0.01 \ldots 0.02$ ), the core bounce is not caused by the stiffening of the EoS but rather by centrifugal forces. However, in contrast to the multiple centrifugal bounce behavior of the (simple hybrid EoS) model A2B4G1 discussed in Sect. 4.1.2, with microphysics only a single bounce with subsequent formation of a quasi-stationary PNS is observed, reflected by a type I waveform. For such cases, where rotation plays a similarly crucial role for the dynamics as e.g. during the disk formation phase in the collapsar model, the potential $\Phi_{\text {Arot }}$ now clearly outperforms potentials $\Phi_{\mathrm{A}}$ and $\Phi_{\mathrm{R}}$ (see Fig. 3). At core bounce we find $\rho_{\text {max,b }}=1.64 \times 10^{14} \mathrm{~g} \mathrm{~cm}^{-3}$ for Case Arot, which is only $2 \%$ larger than the value $1.61 \times 10^{14} \mathrm{~g} \mathrm{~cm}^{-3}$ obtained in the GR simulation, while in Cases A and R the value of $\rho_{\max , \mathrm{b}}$ is underestimated by $27 \%$, and $45 \%$, respectively. In the postbounce phase, however, $\Phi_{\text {Arot }}$ leads to an overestimatate of $\rho_{\max , \mathrm{b}}$ by roughly the same amount as $\Phi_{\mathrm{R}}$ underestimates the correct value. In summary, for this rapidly rotating configuration of a

\footnotetext{
3 Of course, the precise value for the initial rotation parameter beyond which a centrifugal bounce at subnuclear densities occurs also depends on the rotation profile and the choice of $\gamma_{1}$. Dimmelmeier et al. (2007) should be referred to for a detailed analysis.
} 


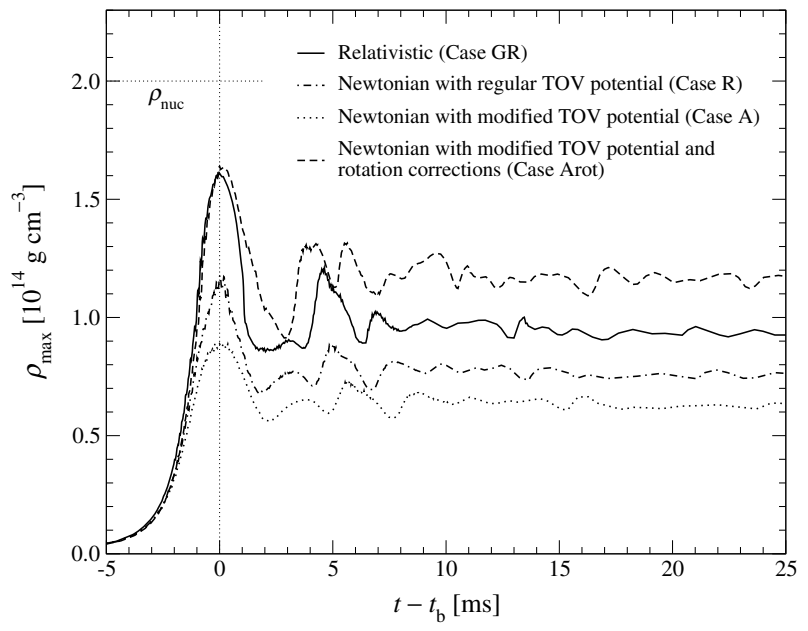

Fig. 3. Same as Fig. 1 but for the centrifugally bouncing microphysical model s20A2B 4 . The effective relativistic potential $\Phi_{\text {Arot }}$ yields very good results around core bounce, but later causes an overestimation of $\rho_{\max }$. In a purely Newtonian simulation this model does not even start to contract.

core that is stabilized against gravity by centrifugal forces rather than pressure gradients, the new effective relativistic potential with rotational corrections performs better than the old potentials at bounce and at least as good after bounce.

We also emphasize that the microphysical model s20A2B4 does not even start to contract in Newtonian gravity due to rotational stabilization. This case where a GR instability leads to the contraction of a core that is still well in the Newtonian limit, clearly illustrates the usefulness of the effective relativistic potential approach. Such a GR instability is also responsible for the collapse of supermassive stars with masses between $10^{4}$ and $10^{8} M_{\odot}$, which are candidates for being progenitors of supermassive black holes. Our results suggest that in this scenario the use of an effective relativistic potential should be able to capture the qualitatively correct collapse dynamics, too, while a purely Newtonian treatment fails.

If a simple hybrid EoS is used, the situation which comes closest to that of the single centrifugal bounce of the microphysical models is when fast initial rotation is combined with a subnuclear adiabatic index $\gamma_{1}$ that is significantly lower than $4 / 3$. Then the collapse of the core is also stopped by only a single bounce (albeit with possibly very strong ring-down oscillations as seen for model A4B2G3 in Fig. 4, or a considerable re-expansion of the core by sometimes more than a factor of 10 in $\rho_{\max }$ as shown for model A4B5G5 in Fig. 5). The post-bounce PNS is either exclusively or predominantly stabilized by centrifugal forces. Many such models have a toroidal density stratification where the off-center maximum density $\rho_{\max }$ exceeds the central density $\rho_{\mathrm{c}}$ by more than one order of magnitude.

In such models the failure of an effective relativistic potential without corrections due to rotation becomes particularly apparent when only a small (possibly off-center) region of the core reaches supranuclear densities and the bounce is caused by a combination of the stiffening of the EoS and by centrifugal forces, as in model A4B2G3 (see Fig. 4). Here, the potential $\Phi_{\mathrm{A}}$ is unable to produce a PNS that is sufficiently compact for the maximum density to remain above $\rho_{\text {nuc }}$, resulting in a post-bounce configuration where $\rho_{\max }$ is $33 \%$ lower than in the GR case. The rotational corrections incorporated into the potential $\Phi_{\text {Arot }}$ on the other hand eliminate this weakness and lead to a final $\rho_{\max }$ which is correct to within $6 \%$.

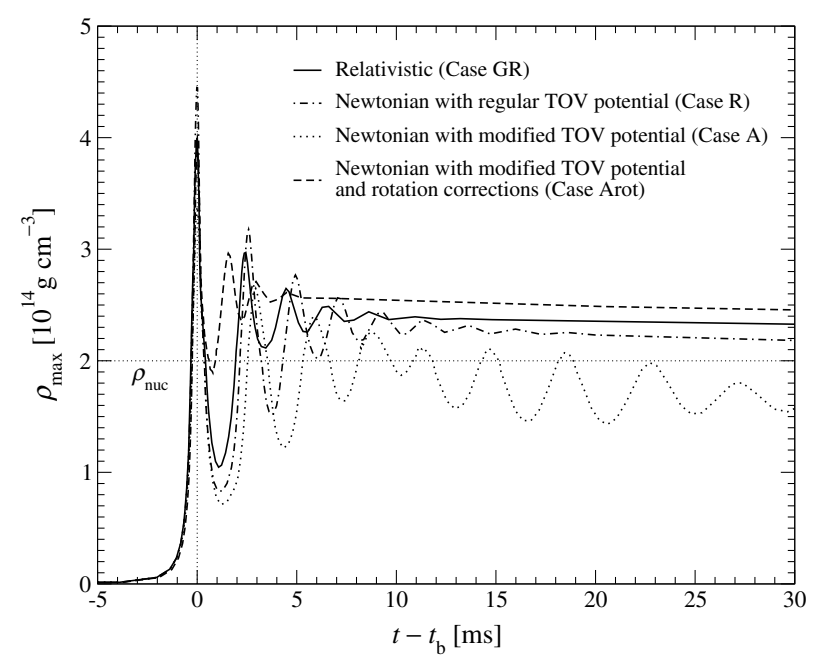

Fig. 4. Same as Fig. 1 but for the rapidly and strongly differentially rotating model A4B2G3. Unlike the effective relativistic potentials $\Phi_{\mathrm{TOV}}$ and $\Phi_{\text {Arot }}$, potential $\Phi_{\mathrm{A}}$ cannot reproduce the correct (supranuclear) maximum density of the core after bounce.

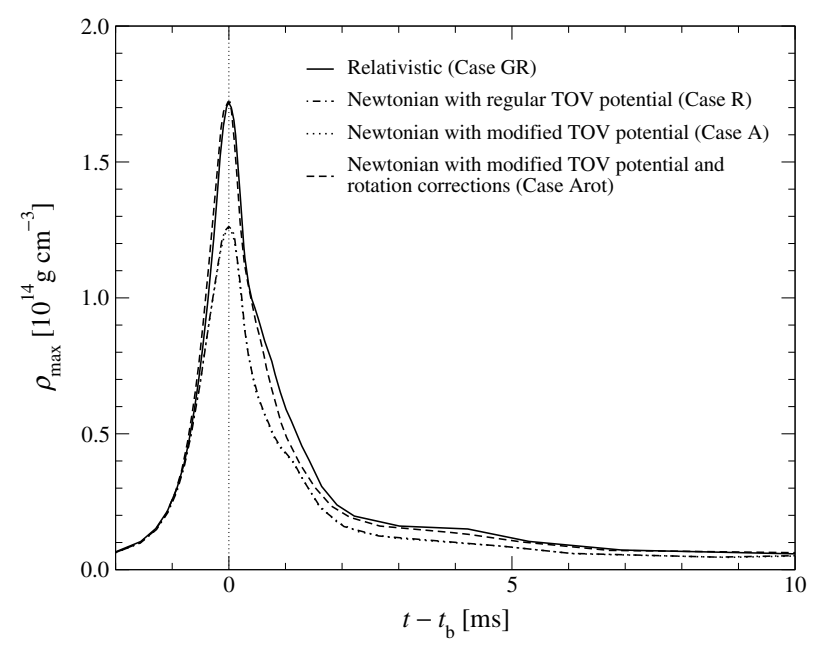

Fig. 5. Same as Fig. 1 but for the single centrifugal bounce model A4B5G5. Again the results obtained with the effective relativistic potential $\Phi_{\text {Arot }}$ show excellent agreement with those found in GR. Note that in this model the density maximum is located off-center, and that the differences between the Case $\mathrm{A}$ and Case $\mathrm{R}$ are minimal.

The results presented in Figs. 4 and 5 demonstrate that for very rapidly rotating models with a simple matter prescription, the new potential $\Phi_{\text {Arot }}$ is superior to $\Phi_{\mathrm{A}}$ both at bounce (showing perfect agreement with the GR results for model A4B5G5) and in the post-bounce phase, while it performs at least equally well as $\Phi_{R}$. We again emphasize that the TOV potential $\Phi_{R}$ does not give the correct results for slow or vanishing rotation, and is thus not as versatile in handling a wide variety of rotation states as $\Phi_{\text {Arot }}$.

In summary, we find that unlike the other effective relativistic potentials, $\Phi_{\text {Arot }}$ reproduces the GR results to within an error of at most $15 \%$ in $\rho_{\max }$ in the entire investigated parameter space of rotation for both simple and microphysical models. The new effective relativistic potential also neither produces an overly compact PNS in spherical symmetry or for slow rotation - like the TOV potential $\Phi_{R}$ - nor does it fail for centrifugal bounces as does $\Phi_{\mathrm{A}}$, and to a smaller extent also $\Phi_{\mathrm{R}}$. 


\subsection{Structure and deformation of proto-neutron stars}

Following Paper I, we do not confine our investigation to local quantities like the maximum density $\rho_{\max }$, but also study the spatial structure of each model by comparing radial profiles obtained in GR with simulations using the new effective relativistic potential with corrections due to rotation.

All effective relativistic potentials discussed in this work as well as in Paper I are constructed from a solution of the TOV equation, which is formulated in Schwarzschild radial coordinates. Therefore, our (otherwise) Newtonian simulations presuppose a specific gauge choice which is different from that used in the GR simulations, where the metric equations in the CFC approximation are based on isotropic radial coordinates. As a consequence, it is necessary to apply a coordinate transformation in order to be able to perform a resonable comparison between both classes of models.

As in Paper I we take the standard Schwarzschild line element in spherical symmetry,

$\mathrm{d} s^{2}=-\alpha^{2} \mathrm{~d} t^{2}+\frac{1}{1-2 m / r} \mathrm{~d} r^{2}+r^{2} \mathrm{~d} \Omega^{2}$,

and replace it by the one in isotropic radial coordinates:

$\mathrm{d} s_{\text {iso }}^{2}=-\alpha^{2} \mathrm{~d} t^{2}+\phi^{4}\left(\mathrm{~d} r_{\text {iso }}^{2}+r_{\text {iso }}^{2} \mathrm{~d} \Omega^{2}\right)$.

For details about this transformation we refer to Appendix B of Paper I, in particular Eq. (B.8).

As long as the shift vector $\beta^{i}$ is close to zero and the deviation of the metric functions $\alpha$ and $\phi$ from spherical symmetry are not too large, $r_{\text {iso }}$ then yields a good estimate for the (in general not spherically symmetric) GR spacetime metric. These conditions are very well satisfied even during the most dynamic phases of the collapse: the radial shift vector component typically reaches a maximum value of the order $\left|\beta_{\mathrm{r}}\right| \approx 0.01$ (in units of the speed of light $c$ ) near bounce and then decreases as the PNS settles down to a quasi-stationary state, while the meridional component $\beta_{\theta}$ is even smaller. The rotational component $\beta_{\varphi}$ is about one order of magnitude smaller than the maximum rotational velocity $v_{\varphi}$, i.e. its absolute value rarely exceeds 0.01 even for the most rapidly rotating models. Moreover, the anisotropy of the metric functions $\alpha$ and $\phi$ is significantly smaller than the anisotropy of the matter fields.

The potentials $\Phi_{\mathrm{A}}$ and $\Phi_{\text {Arot }}$ are identical in the non-rotating limit and differ only slightly for slow rotation, as the corrections due to rotation are negligible. Thus, we concentrate our discussion on the two rapidly rotating models A4B2G3 and A4B5G5. Furthermore, we investigate only the innermost regions of the collapsed stellar core constituting the nascent PNS, which is in approximate equilibrium after the core bounce and the ringdown phase.

Figures 6 and 8 illustrate that the radial profiles of the density $\rho$ and the specific internal energy $\epsilon$ in Case Arot are in very good agreement with those obtained in GR for both models. To demonstrate that this excellent matching is independent of the angular location, we show profiles of $\rho$ at three different latitudes - along the polar axis, at $\theta=\pi / 4$, and in the equatorial plane, respectively. For model A4B2G3, in both cases (Arot and GR) we observe after the ring-down phase a similar toroidal structure with a maximum density of $\rho_{\max } \approx(2.3-2.4) \times 10^{14} \mathrm{~g} \mathrm{~cm}^{-3}$ at a radius of 5-6 km in the equatorial plane, and a central density $\rho_{\mathrm{c}}$ close to $1.0 \times 10^{14} \mathrm{~g} \mathrm{~cm}^{-3}$. Even for model A4B5G5, which develops a very extreme toroidal structure and is depicted in the highly dynamic phase during core bounce in the lower panel of
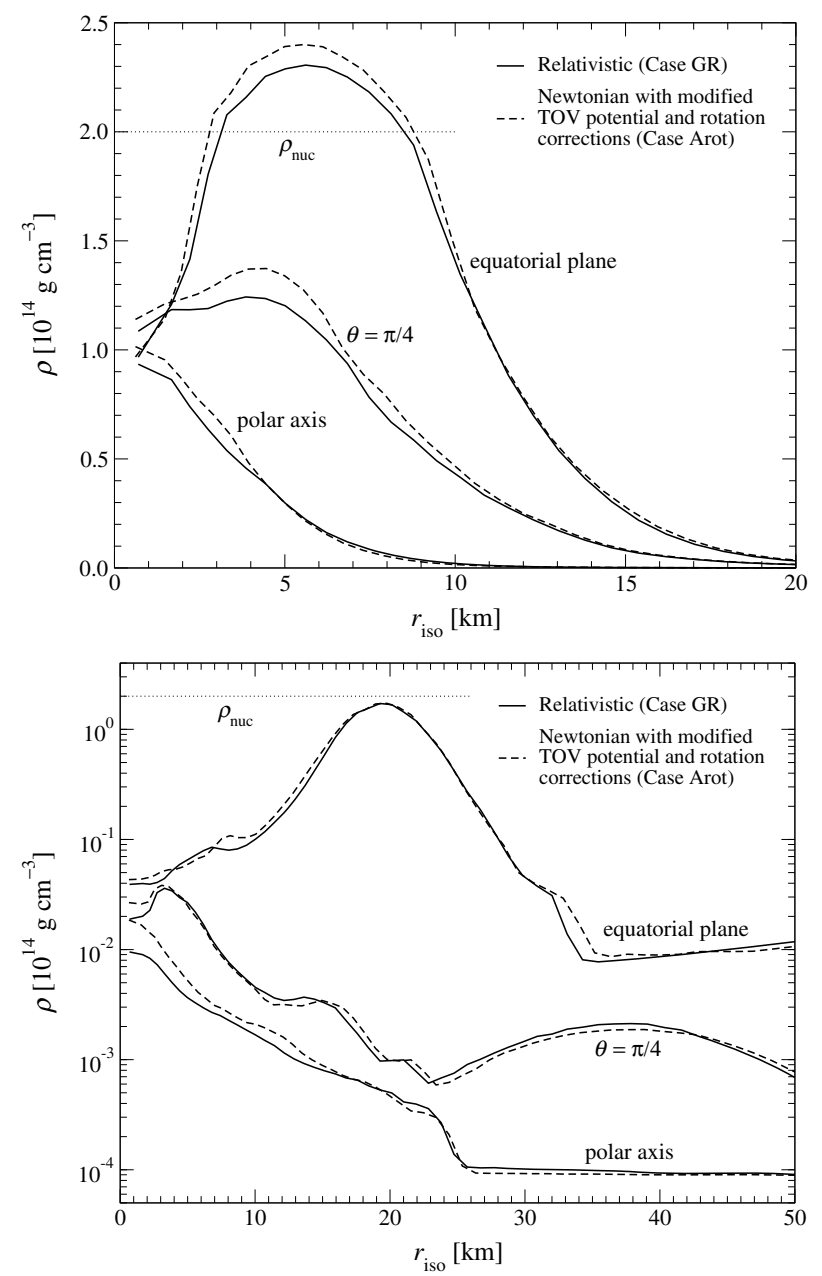

Fig. 6. Radial profiles of the density $\rho$ for model A4B2G3 (top panel; $40 \mathrm{~ms}$ after bounce) and model A4B5G5 (bottom panel, at the time of bounce) at various polar angles for Case GR (solid lines) and Case Arot (dashed lines), respectively. For the potential $\Phi_{\text {Arot }}$ we apply a transformation to the isotropic radial coordinate (see text for details). Both models rotate rapidly and strongly differentially, and model A4B5B5 bounces at subnuclear densities.

Fig. 6, the new potential $\Phi_{\text {Arot }}$ yields results which closely match those in GR. The maximum density $\rho_{\max }$ is reached at $r \approx 20 \mathrm{~km}$ in the equatorial plane. Figure 7 shows density contours for this model down to densities of $10^{10} \mathrm{~g} \mathrm{~cm}^{-3}$, and demonstrates how closely Case Arot reproduces the spatial structure of that model in full GR. The step-like features in $\epsilon$ in the low-density regions, which are caused by shock heating, are also reproduced quite accurately, as can be seen in Fig. 8.

Due to the very good agreement of the density and internal energy profiles between Cases GR and Arot already during the dynamical phase of the core bounce we also infer that the use of the transformation from Schwarzschild to isotropic radial coordinates needs not be limited to the case discussed in Paper I, i.e. a spherical PNS in the late post-bounce phase, but works even for strongly rotating and significantly aspherical configurations.

\subsection{Structure of rotating neutron stars}

The core collapse simulations presented here are limited to a maximum compactness of the PNS of $2 M_{\mathrm{PNS}} / R_{\mathrm{PNS}} \sim 0.2$ in the early post-bounce phase, where $M_{\mathrm{PNS}}$ and $R_{\mathrm{PNS}}$ are the mass and radius of the PNS, respectively. In order to extend the assessment 


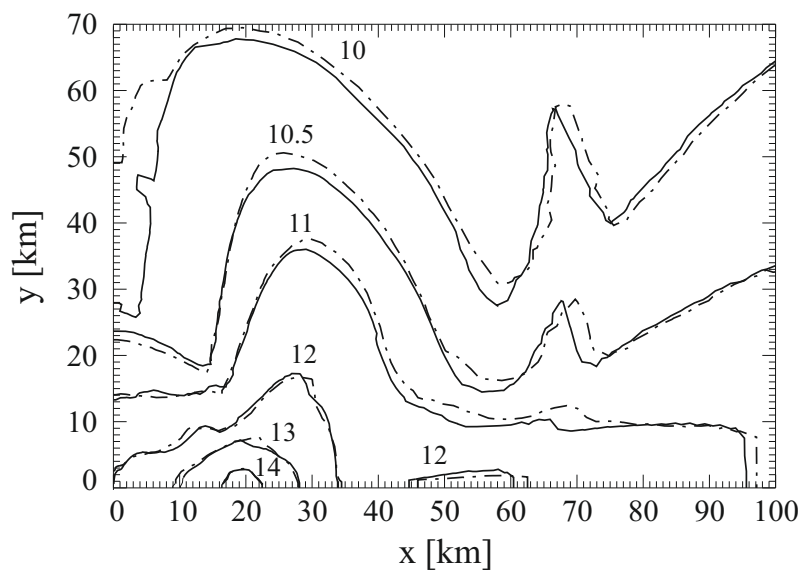

Fig. 7. Density contours for model A4B5G5 at bounce in full GR (solid lines), and for Case Arot (dashed-dotted lines). $\log _{10} \rho$ is indicated by the contour labels. As in Fig. 6 we apply a transformation to isotropic radial coordinates.
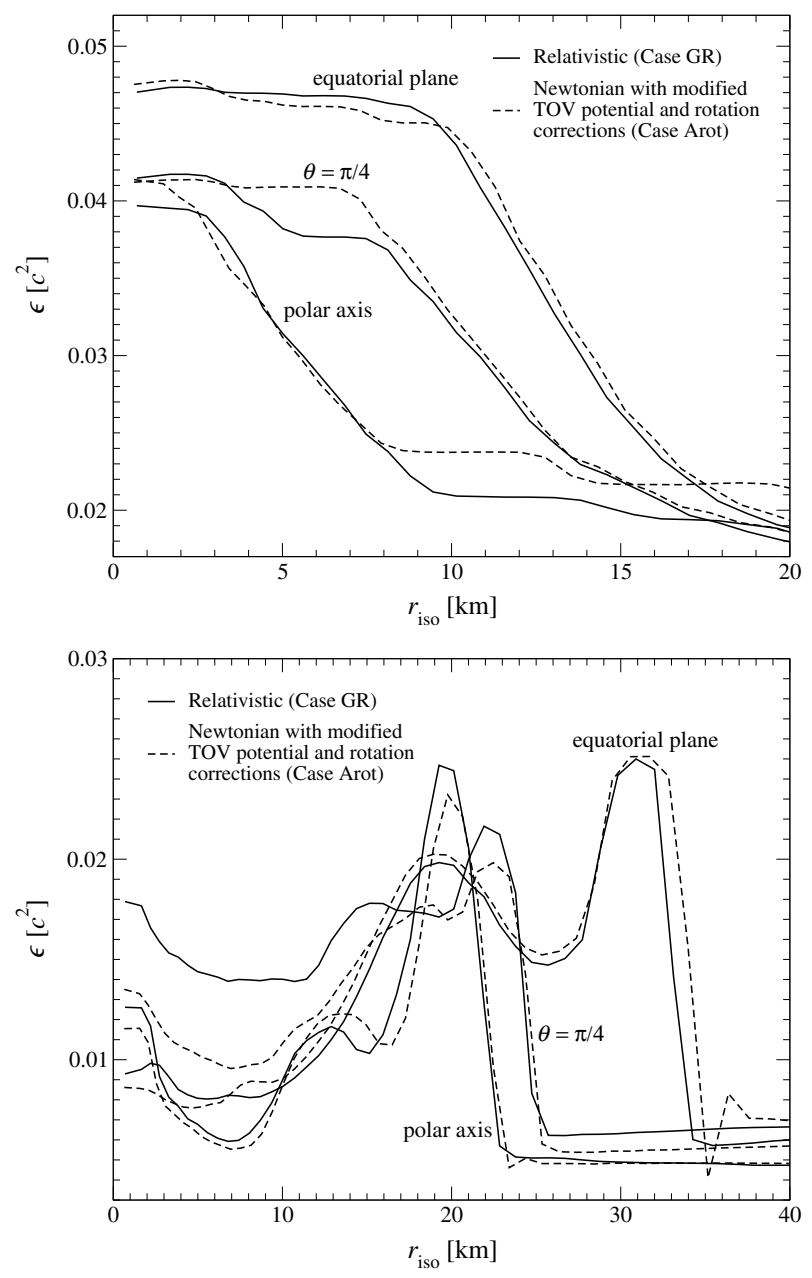

Fig. 8. Same as Fig. 6, but for the specific internal energy $\epsilon$. Note that potential $\Phi_{\text {Arot }}$ (dashed lines) closely reproduces the step-like features in $\epsilon$ of the model in GR (solid lines).

of the quality of a Newtonian simulation with an effective relativistic potential to a more strongly relativistic regime, we also investigate equilibrium models of a rotating NS using the polytropic EoS (27). These can serve as an approximation to either a cooling PNS which has already evolved over several $100 \mathrm{~ms}$ (and cannot be directly simulated with our code due to

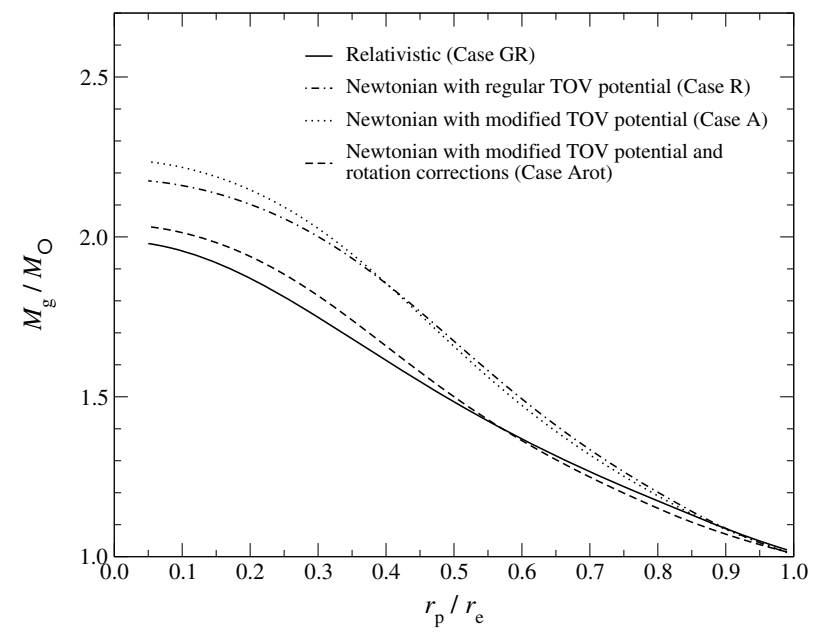

Fig. 9. Dependence of the gravitational mass $M_{\mathrm{g}}$ on the axis ratio $r_{\mathrm{p}} / r_{\mathrm{e}}$ for rotating NS models with a maximum density $\rho_{\max }=3.95 \times$ $10^{14} \mathrm{~g} \mathrm{~cm}^{-3}$, and a differential rotation parameter $\hat{A}=0.3$. We show the results for Case GR (solid line), Case R (dashed-dotted line), Case A (dotted line), and Case Arot (dashed line), respectively. The results obtained with the effective relativistic potential $\Phi_{\text {Arot }}$ including corrections due to rotation agree best with the ones of GR.

computational constraints and the missing necessary microphysics) or a cold NS. Such models also enable us to check the reliability of our approach for scenarios such as accretioninduced collapse. Similarly, the ability to correctly handle equilibrium models of compact objects with the effective relativistic potential approach is a prerequisite for investigating instabilities or oscillations in a NS.

Therefore, we also perform an analysis of various rotating NS models in GR and for the different effective relativistic potentials. We find that the new potential $\Phi_{\text {Arot }}$ reproduces quite closely the correct GR dependence of most global quantities (like gravitational mass, binding energy, etc.) on e.g. the axis ratio of the NS, even for strong and differential rotation, and significant toroidal deformation.

As long as the NS is not too compact (i.e. $\rho_{\mathrm{c}}<4 \times$ $10^{14} \mathrm{~g} \mathrm{~cm}^{-3}$, which are typical values for a PNS during the first few $100 \mathrm{~ms}$ after core bounce), the results for Case R and A do not differ strongly. This is evident from Fig. 9, where we plot the gravitational mass $M_{\mathrm{g}}$ for a sequence of NS models with constant central density $\rho_{\mathrm{c}}=3.95 \times 10^{14} \mathrm{~g} \mathrm{~cm}^{-3}$ and differential rotation parameter $\hat{A}=0.3$ (where $\hat{A}=A / r_{\mathrm{e}}$ ), and vary the axis ratio $r_{\mathrm{p}} / r_{\mathrm{e}}$. Here $r_{\mathrm{p}}$ and $r_{\mathrm{e}}$ are the polar and equatorial radius of the NS, respectively. Figure 9 shows that even for an extreme axis ratio $r_{\mathrm{p}} / r_{\mathrm{e}}=0.05$, the deviation of $M_{\mathrm{g}}$ from its GR value is only about $15 \%$ for the worst potential (Case A), and much better for potential $\Phi_{\text {Arot }}$. The gravitational binding energy $W$ is somewhat more sensitive to the inclusion of relativistic effects; we obtain a considerably larger value of $|W|=6.01 \times 10^{53} \mathrm{erg}$ for Case A compared to $|W|=4.60 \times 10^{53} \mathrm{erg}$ in full GR $(+31 \%)$. However, using the potentials $\Phi_{\mathrm{TOV}}$ or $\Phi_{\text {Arot }}$ we obtain $|W|=5.31 \times 10^{53} \mathrm{erg}(+15 \%)$ and $|W|=5.04 \times 10^{53} \mathrm{erg}(+10 \%)$, which is reasonably close to the GR value. We also find good agreement for quantities like the baryonic mass, and the central angular velocity, as well as for the spatial structure of the NS models (e.g. polar and equatorial radii, density profiles).

For more compact configurations, we find the deviations from the GR models to be more pronounced, as illustrated by Fig. 10, where we show $M_{\mathrm{g}}$ as a function of the maximum 


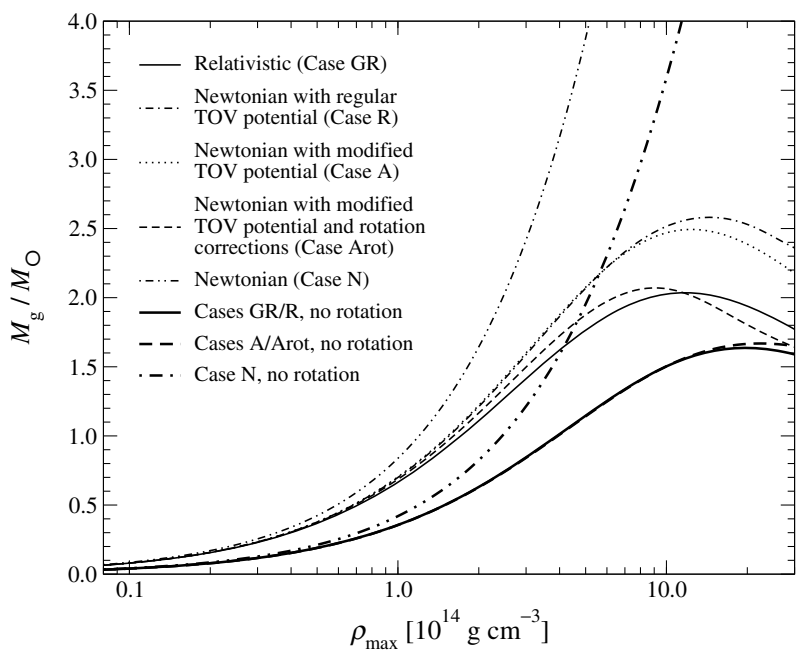

Fig. 10. Same as Fig. 9, but showing the dependence of the gravitational mass $M_{\mathrm{g}}$ on the maximum mass $\rho_{\max }$ for rotating NS models with an axis ratio $r_{\mathrm{p}} / r_{\mathrm{e}}=0.4$ and a differential rotation parameter $\hat{A}=0.3$. Again the results obtained with the effective relativistic potential $\Phi_{\text {Arot }}$ agree best with those in GR, while in the purely Newtonian case the values for $M_{\mathrm{g}}$ are consistently too high. Without rotation (thick lines) the Cases GR and R, as well as A and Arot are identical, respectively.

density $\rho_{\max }$ for $\hat{A}=0.3$ and an axis ratio $r_{\mathrm{p}} / r_{\mathrm{e}}=0.4$. Apparently the error in $M_{\mathrm{g}}$ reaches $0.6 M_{\odot}$ for potential $\Phi_{\mathrm{A}}$, and $0.47 M_{\odot}$ for potential $\Phi_{\mathrm{TOV}}$, but does not exceed $0.15 M_{\odot}$ for the new potential $\Phi_{\text {Arot }}$ with rotational corrections.

It is also noteworthy that for fast rotation the deviations from GR in Cases $\mathrm{A}$ and $\mathrm{R}$ already manifest themselves well below the density $\rho_{\max } \approx 5 \times 10^{14} \mathrm{~g} \mathrm{~cm}^{-3}$ of maximum gravitational mass, which is the transition point to the unstable branch. For non-rotating models the deviations remain small even beyond that point, which is then located at $\rho_{\max } \approx 20 \times 10^{14} \mathrm{~g} \mathrm{~cm}^{-3}$ (see Fig. 10, and cp. Paper I). On the other hand, the purely Newtonian results overestimate $M_{\mathrm{g}}$ by a factor of 2 already for $\rho_{\text {max }}=8 \times 10^{14} \mathrm{~g} \mathrm{~cm}^{-3}$, regardless of the strength of rotation. Thus, the effective relativistic potentials, and particularly the new one $\Phi_{\text {Arot }}$ give a clear improvement over a purely Newtonian treatment. As for the core collapse simulations, Case Arot is very robust and accurate over the entire range of rotation states with varying $\hat{A}$ and $r_{\mathrm{p}} / r_{\mathrm{e}}$. It exhibits a maximum error in $M_{\mathrm{g}}$ of $\approx 10 \%$ compared to more than $20 \%$ and $30 \%$ for Case R and Case A, respectively.

\subsection{Oscillations of neutron stars}

The effective relativistic potential approach obviously approximates very well the collapse dynamics of rapidly rotating stellar cores as compared to a simulation in GR. Furthermore, it also gives accurate results for the structure of a nascent PNS after core collapse as well as of a rotating cold NS. We now subject this approach to an even more stringent test for its applicability to the post-bounce evolution of a PNS or to instability problems in a rotating NS. To this end, we consider the dynamics of a NS near equilibrium and oscillations in the linear regime. For convenience, we restrict the study to non-rotating models (where Case A and Arot are identical), as the results discussed here are qualitatively independent of rotation.

We first evolve a very compact polytropic $\gamma=2 \mathrm{NS}$ model with $\rho_{\mathrm{c}}=7.9 \times 10^{14} \mathrm{~g} \mathrm{~cm}^{-3}$, which is a density value typically reached around $1 \mathrm{~s}$ after core bounce. When applying a small

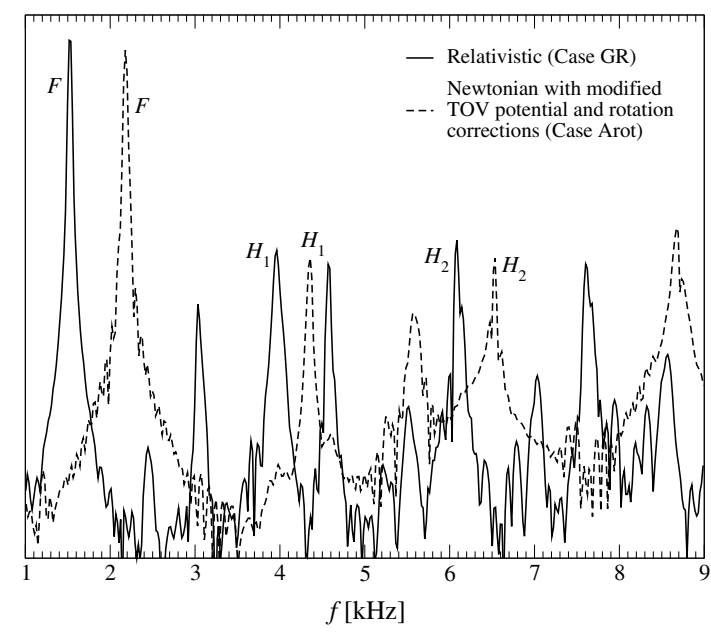

Fig. 11. Power spectrum of the time evolution of the maximum density $\rho_{\max }$ for a very compact non-rotating NS model for Case GR (solid line) and Case Arot (dashed line), respectively. The $F$-mode and its harmonics $H_{1}$ and $H_{2}$ are clearly visible. For all investigated modes the frequencies obtained with the potential $\Phi_{\text {Arot }}$ are significantly higher than those in GR.

radial velocity perturbation to excite radial normal modes, we find significantly different frequencies of the eigenmodes as compared to GR if we use any of the effective relativistic potentials discussed here. For instance, all these potentials overestimate the frequency of the fundamental radial $F$-mode by about $45 \%$. This can be clearly seen in Fig. 11, where we present the power spectrum of the time evolution of $\rho_{\max }$, in which the eigenmodes can be identified as distinct peaks.

For a less compact configuration with $\rho_{\mathrm{c}}=3.95 \times$ $10^{14} \mathrm{~g} \mathrm{~cm}^{-3}$, which is obtained a few $100 \mathrm{~ms}$ after bounce, the error is somewhat smaller $(\sim 22 \%)$ but still much larger than what could be expected given the excellent agreement in the structure of the NS model between the GR case and the use of an effective relativistic potential. In addition, the higher harmonics of the $F$-mode like the $H_{1}$-mode or the $H_{2}$-mode also exhibit this apparent discrepancy (see Fig. 11).

The failure of the effective relativistic potential method in the context of NS oscillations can be understood by comparing the equations of hydrodynamics in GR and the Newtonian formulation ${ }^{4}$. Limiting ourselves to the most straightforward case of the TOV potential in Case $\mathrm{R}$ and to spherical symmetry, we find the following evolution equation for the radial velocity:

$\frac{\partial v_{\mathrm{r}}}{\partial t}=-\frac{1}{\rho} \frac{\partial P}{\partial r}-\frac{\partial \bar{\Phi}_{\mathrm{TOV}}}{\partial r}$.

An analogous equation can be obtained in GR, starting from the formulation of van Riper (1979), which uses Schwarzschild coordinates like in the case of the TOV potential. After rearranging some terms to allow direct identifications with Case $\mathrm{R}$, one arrives at

$\frac{\partial\left(\alpha^{-1} v_{\mathrm{r}}\right)}{\partial t}=\frac{\alpha}{h}\left(-\frac{1}{\rho} \frac{\partial P}{\partial r}-\frac{\partial \bar{\Phi}_{\mathrm{TOV}}}{\partial r}\right)$

Unlike the Newtonian Eq. (34), the GR Eq. (35) contains an additional factor given by the ratio of the lapse function $\alpha$ and the

\footnotetext{
4 We remind the reader here that except for changes in the gravitational potential source terms, in the effective relativistic potential approach the equations of Newtonian hydrodynamics remain unaltered.
} 
specific relativistic enthalpy $h$. For a stationary state, the left hand sides vanish and both equations become equivalent. This explains why potential $\Phi_{\mathrm{TOV}}$ (or other modified potentials like $\Phi_{\mathrm{A}}$ and $\Phi_{\text {Arot }}$ ) can be successfully applied to model the structure of PNS or NS models.

On the other hand, in the general dynamic case, the additional factor cannot be discarded, and will, for example, be present in the linearized perturbation equations. Since the lapse function $\alpha$ is significantly below 1 in compact NS (with a central value of $\alpha_{\mathrm{c}} \approx 0.7$ ), this factor has a considerable impact on the linearized perturbation equations and consequently on the eigenfrequency spectrum. For special cases, such as spherical pulsations, it may still be possible to obtain simple scaling relations for some of the effective relativistic potentials, but this is beyond the scope of this work. At present, however, we conclude that the effective relativistic potential approach is much less suited for studying the frequencies of NS oscillations than for approximating e.g. the structure or global quantities like mass and radius. It is conceivable that a pseudo-Newtonian treatment should be used with caution whenever NS or PNS oscillations play a crucial dynamical role, e.g. in the acoustic explosion mechanism suggested by Burrows et al. (2006), whose potential is still disputed (Weinberg \& Quataert 2008). A fortiori, this also applies for a purely Newtonian approach. A fully relativistic treatment of the hydrodynamics would probably be desirable in such a context to obtain firm quantitative predictions, even though it may not be a necessary ingredient for a qualitatively correct model.

\subsection{Improvement of the gravitational wave extraction}

After having investigated and compared the quality of the new potential $\Phi_{\text {Arot }}$, we now focus on the issue of GW emission from simulations of rotational stellar core collapse using the effective relativistic potential approach. In our simulations, we compute the radiation quadrupole moment $A_{20}^{\mathrm{E} 2}$ according to the first timeintegrated Newtonian quadrupole formula (Finn 1989; Blanchet et al. 1990),

$$
\begin{aligned}
A_{20}^{\mathrm{E} 2}= & \frac{32 \pi^{3 / 2}}{\sqrt{15}} \frac{\mathrm{d}}{\mathrm{d} t} \int r^{3} \sin \theta \mathrm{d} \theta \mathrm{d} r \\
& \times \rho\left[v_{\mathrm{r}}\left(3 \cos ^{2} \theta-1\right)-3 v_{\theta} \sin \theta \cos \theta\right] .
\end{aligned}
$$

We restrict the integration to those grid cells separated from the initial low-density atmosphere by at least five radial zones, thus suppressing numerical noise due to the reassignment of cells to the atmosphere. The amplitude $A_{20}^{\mathrm{E} 2}$ is related to the dimensionless GW strain $h$ in the equatorial plane at a distance $r$ to the source by (see e.g. Dimmelmeier et al. 2002b)

$h=\frac{1}{8} \sqrt{\frac{15}{\pi}} \frac{A_{20}^{\mathrm{E} 2}}{r}=8.85 \times 10^{-21} \frac{A_{20}^{\mathrm{E} 2}}{10^{3} \mathrm{~cm}} \frac{10 \mathrm{kpc}}{r}$.

Obergaulinger et al. (2006) presented a comparison of two core collapse models both simulated in GR and using the TOV potential of Case R. They found that the simulations with the TOV potential lead to an error of about $50 \%$ in the GW peak amplitude $\left|A_{20}^{\mathrm{E} 2}\right|_{\text {max }}$ (that is reached around bounce) for model A1B3G3 despite the fact that e.g. the evolution of the $\rho_{\max }$ is quite similar in both cases. This is confirmed by our simulations of a large and representative set of stellar core collapse models both with a simple matter model and also with more detailed microphysics.

For slowly rotating models, where hydrodynamic quantities like $\rho_{\max }$, density profiles, etc. obtained using an effective relativistic potential agree with those in GR very well, the errors
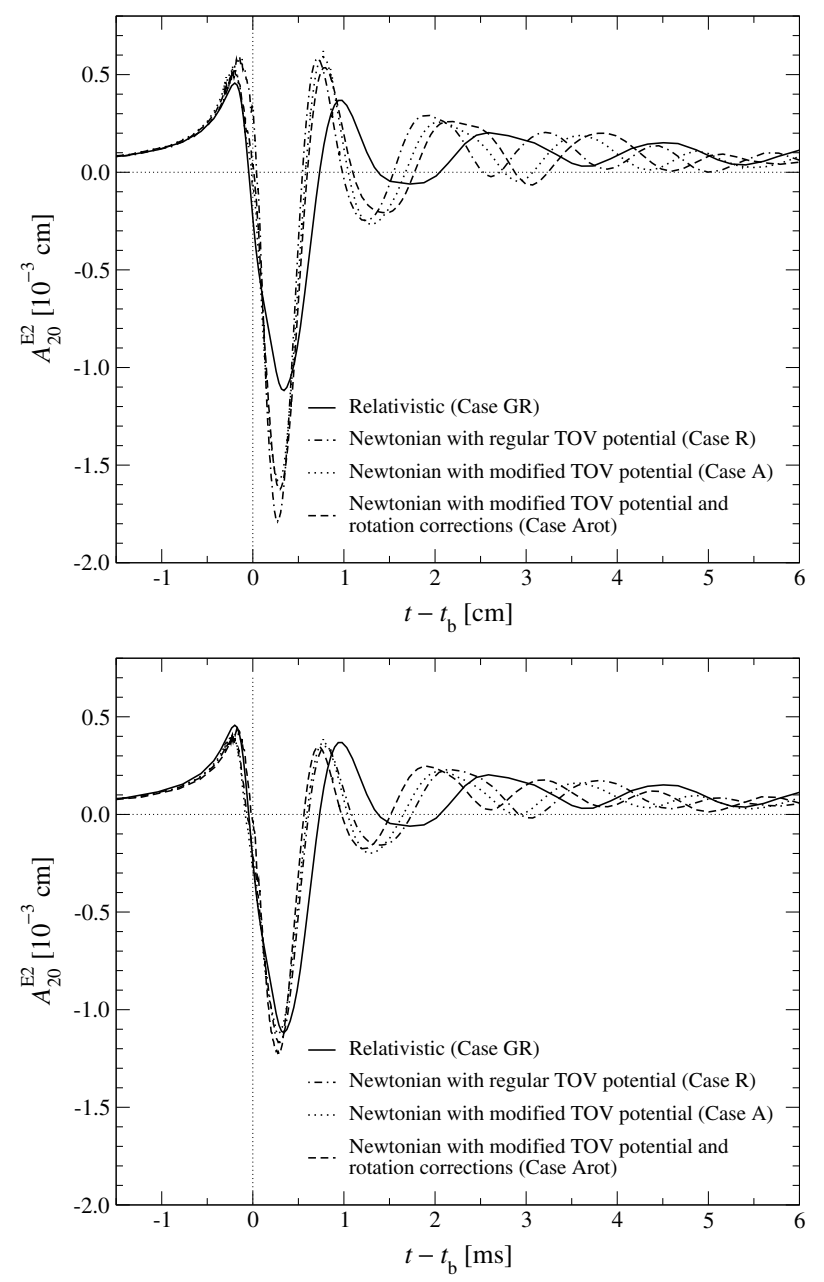

Fig. 12. Time evolution of the GW amplitude $A_{20}^{\mathrm{E} 2}$ for model A1B1G1 extracted from a simulation in Case GR (solid), Case R (dashed-dotted), Case A (dotted), and Case Arot (dashed), respectively, using the timeintegrated quadrupole formula. The top panel shows the waveforms obtained without the transformation to the isotropic radial coordinate for the simulations with an effective relativistic potential. If the transformation is included, the difference in the GW amplitudes compared to Case GR is reduced considerably (lower panel).

in $\left|A_{20}^{\mathrm{E} 2}\right|_{\max }$ (and the entire waveform) are significant, often between $30 \%$ and $50 \%$. This is exemplified by model A1B1G1, whose waveform is shown in the top panel of Fig. 12.

The quadrupole formula is derived in the Newtonian weakfield and low-velocity limit, and has to be generalized to a GR spacetime before a better matching to GR results can be expected. This requires a (non-unique) choice of the radial coordinate. In the CFC metric of Case GR, isotropic radial coordinates are assumed. On the other hand, as already detailed in Sect. 4.2, the effective relativistic potentials are derived from the TOV solution in Schwarzschild radial coordinates. The analogy to the discussion of the spatial structure of the PNS suggests that consistency between GR and Newtonian-based simulations requires the use of the same gauge and coordinate choice for any comparison of GW amplitudes.

We therefore apply the transformation from the Schwarzschild radial coordinate to the isotropic radial coordinate (see Eq. (B.8) in Paper I) when extracting GW signals via the quadrupole formula. This transformation effectively reduces the magnitude of the factor $r^{3}$ in Eq. (36) and thus leads to a considerably lower amplitude, while the waveform 
Table 3. Absolute value of the GW peak amplitude $\left|A_{20}^{\mathrm{E} 2}\right|_{\max }$ for all investigated core collapse models in Case GR, or evolved with the effective relativistic potential of Case R, A, and Arot, respectively. For each potential, the relative deviation from Case GR (in percent) is given in parentheses.

\begin{tabular}{|c|c|c|c|c|c|c|c|}
\hline \multirow[b]{2}{*}{ Model } & \multirow[b]{2}{*}{ Case GR } & \multicolumn{3}{|c|}{ no radial coordinate transformation } & \multicolumn{3}{|c|}{ transformation to isotropic radial coordinate } \\
\hline & & Case $\mathrm{R}$ & Case A & Case Arot & Case $\mathrm{R}$ & Case A & Case Arot \\
\hline A1B1G1 & 1128 & $1789(+58)$ & $1581(+40)$ & $1627(+44)$ & $1227(+9)$ & $1170(+4)$ & $1127( \pm 0)$ \\
\hline A1B2G1 & 2153 & $3057(+42)$ & $2767(+29)$ & $2861(+33)$ & $2062(-4)$ & $1955(+9)$ & $1980(-8)$ \\
\hline A1B3G1 & 3340 & $4242(+27)$ & $3588(+7)$ & $4239(+27)$ & $2871(-14)$ & $2595(-22)$ & $2858(-15)$ \\
\hline A1B3G2 & 2094 & $2767(+32)$ & $2508(+20)$ & $2736(+31)$ & $1956(-5)$ & $1838(-12)$ & $1938(-15)$ \\
\hline A1B3G3 & 778 & $1104(+42)$ & $1049(+35)$ & $1055(+36)$ & $837(+8)$ & $810(+4)$ & $801(-7)$ \\
\hline A2B4G1 & 651 & $630(-3)$ & $615(-6)$ & $665(+2)$ & $572(-12)$ & $562(-14)$ & $604(-7)$ \\
\hline $\begin{array}{l}\text { A3B1G1 } \\
\text { A3B2G4 } \\
\text { A3B4G2 }\end{array}$ & $\begin{array}{r}2456 \\
598 \\
842\end{array}$ & $\begin{array}{c}3711(+51) \\
791(+32) \\
802(-5)\end{array}$ & $\begin{array}{c}3238(+32) \\
762(+27) \\
775(-8)\end{array}$ & $\begin{array}{c}3372(+37) \\
779(+30) \\
900(+7)\end{array}$ & $\begin{array}{c}2367(-4) \\
623(+4) \\
731(-13)\end{array}$ & $\begin{array}{c}2187(-11) \\
606(+1) \\
705(-16)\end{array}$ & $\begin{array}{c}2213(-10) \\
608(+2) \\
790(-6)\end{array}$ \\
\hline $\begin{array}{l}\text { A4B1G1 } \\
\text { A4B1G2 } \\
\text { A4B2G2 } \\
\text { A4B2G3 } \\
\text { A4B5G4 } \\
\text { A4B5G5 }\end{array}$ & $\begin{array}{l}3269 \\
2745 \\
4490 \\
3093 \\
2466 \\
3383\end{array}$ & $\begin{array}{l}4610(+41) \\
3593(+31) \\
5067(+13) \\
3546(+15) \\
2754(+12) \\
3766(+11)\end{array}$ & $\begin{array}{l}3746(+15) \\
3041(+11) \\
4393(+2) \\
3032(-2) \\
2725(+11) \\
3744(+11)\end{array}$ & $\begin{array}{l}4384(+34) \\
3550(+29) \\
5704(+27) \\
3823(+24) \\
2669(+8) \\
3614(+7)\end{array}$ & $\begin{array}{l}2836(-13) \\
2382(-13) \\
3444(-23) \\
2597(-16) \\
2505(+2) \\
3476(+3)\end{array}$ & $\begin{array}{l}2485(-24) \\
2103(-23) \\
3084(-31) \\
2407(-22) \\
2479(+1) \\
3457(+2)\end{array}$ & $\begin{array}{l}2652(-19) \\
2284(-17) \\
3581(-20) \\
2606(-16) \\
2345(-5) \\
3258(-4)\end{array}$ \\
\hline $\begin{array}{l}\text { s20A1B1 } \\
\text { s20A2B4 }\end{array}$ & $\begin{array}{r}69 \\
339\end{array}$ & $\begin{array}{l}108(+57) \\
330(-3)\end{array}$ & $\begin{array}{l}101(+46) \\
310(-9)\end{array}$ & $\begin{array}{r}89(+29) \\
353(+4)\end{array}$ & $\begin{array}{l}- \\
-\end{array}$ & $\begin{array}{l}- \\
-\end{array}$ & $\begin{array}{r}68(-1) \\
321(-5)\end{array}$ \\
\hline
\end{tabular}

shape itself remains essentially unchanged. In many cases, the coordinate transformation significantly improves the agreement with the GR results: for the slow and almost uniform rotator A1B1G1 (see lower panel of Fig. 12), $A_{20}^{\mathrm{E} 2}$ extracted from a simulation with any of the effective relativistic potentials now deviates by less than $10 \%$ from the GR simulation, as opposed to more than $40 \%$ without the coordinate change. For the microphysical model s20A1B1, which has the same initial density profile and rotation rate, the effect is also dramatic: the deviation is reduced from $29 \%$ to $1 \%$ for Case Arot.

We observe a similar improvement for most other investigated models with slow and weakly differential rotation, i.e. where $T /|W| \lesssim 0.1$ during the whole evolution: Table 3 shows that for the potentials $\Phi_{\mathrm{R}}$ and $\Phi_{\text {Arot }}$ the deviation from GR is reduced in such cases (all the $A 1$ and $B 1$ models), and there are only three exceptions for potential $\Phi_{\mathrm{A}}$. Even for fast rotators (see e.g. the B3, B4, and B5 models in Table 3), we observe no errors in the bounce signal larger than 20\% (Case Arot) and $24 \%$ (Case R), respectively, when the coordinate transformation is applied. For more rapid rotators, Case A is an exception as the transformation actually degrades the quality of the results. This is to be expected, as that potential often leads to a core density (i.e. compactness) at bounce that is significantly below the value obtained with potential $\Phi_{\mathrm{R}}$ (which always gives larger densities than potential $\Phi_{\mathrm{A}}$ ) and potential $\Phi_{\text {Arot }}$ (which includes corrections due to rotation).

In summary we can infer that the combination of the potential $\Phi_{\text {Arot }}$ and the radial coordinate transformation in the GW extraction based on the quadrupole formula delivers waveforms that are very accurate when compared to the ones from GR simulations for a wide range of rotation rates and profiles of collapsing compact objects.

\section{Summary and conclusions}

We have compared pseudo-Newtonian simulations of rotational stellar core collapse with three different effective relativistic gravitational potentials to reference simulations with the conformal flatness approximation of general relativity. This has been done using either a simplified EoS or a microphysical treatment of matter. We have been able to improve the effective relativistic potential approach used by Marek et al. (2005) and Obergaulinger et al. (2006) both in the regime of slow and rapid rotation. Our tests also allow us to assess the applicability of the different old potentials as well as the new improvement in these two regimes.

In the case of slow and moderate rotation, all potentials give good results for the dynamical evolution. This implies that the generic scenario of core collapse in massive stars can probably be treated without including any rotational corrections like implemented in the new potential $\Phi_{\text {Arot }}$, because the cores of these stars are not expected to spin rapidly (Heger et al. 2000). Moreover, the multiple bounce scenario, which is most problematic for the rotationally uncorrected potentials $\Phi_{A}$ and $\Phi_{R}$, is suppressed once deleptonization is taken into account. However, we have demonstrated that the gravitational wave signal can still be significantly improved also in these cases by applying a simple coordinate transformation when utilizing the Newtonian quadrupole formula. Our new effective relativistic potential now yields wave amplitudes which are within $20 \%$ of the values found in relativistic simulations, as opposed to errors of more than $50 \%$ reported by Obergaulinger et al. (2006). In particular, we observe excellent agreement for the most slowly rotating models considered, i.e. the ones closest to the predictions of Heger et al. (2000) in terms of rotation rates. The best overall match with relativistic gravitational wave signals has been obtained with $\Phi_{\text {Arot }}$, which is identical to $\Phi_{\mathrm{A}}$ in spherical symmetry. As in this limit $\Phi_{\mathrm{A}}$ is superior to the original effective relativistic potential $\Phi_{\mathrm{R}}$ (Marek et al. 2005), the potential $\Phi_{\text {Arot }}$ is already a very attractive choice in the regime of slow rotation.

For strong rotation we have studied more demanding test problems for our potentials than considered in Paper I. In order to assess in general the usefulness of the effective relativistic potential approach for prospective simulations of rotational instabilities in a proto-neutron star or a cold neutron star, or for the accretion-induced collapse scenario, we have investigated models of a rotating neutron star, and rotational stellar core collapse with high rotation rates and an extremely differential angular 
momentum distribution. We have included very rapidly rotating core collapse models for which $T /|W| \approx 0.4$ is reached, and have also discussed equilibrium models of a neutron star with strong differential rotation and axis ratios down to $r_{\mathrm{p}} / r_{\mathrm{e}}=0.05$. The rotational parameters of these models were chosen such as to also provide a reasonable description of the accretion-induced collapse scenario, which has however not been directly simulated in this work.

As in Paper I we confirm that the accuracy of Cases R and A declines for rapidly rotating configurations. For instance, these potentials lead to a gross underestimation of the maximum density at bounce for core collapse models with a single centrifugal bounce, such as model A4B5G5, where the deviation to the general relativistic results is as large as $27 \%$. These "old" potentials also cannot reproduce the structure of a rapidly rotating neutron star, although they still provide a clear improvement over the purely Newtonian treatment. By including rotational corrections in our new potential $\Phi_{\text {Arot }}$, we have been able to obtain much closer agreement with the general relativistic results in many core collapse simulations as well as for rapidly rotating neutron star models in equilibrium. In Case Arot the maximum density at bounce, for example, now always lies within $10 \%$ of the correct value, and radial profiles of strongly rotating and toroidally deformed models also agree very well with the ones from general relativistic simulations. Thus, in contrast to potentials $\Phi_{R}$ and $\Phi_{\mathrm{A}}$, we infer that for all practical purposes the new potential $\Phi_{\text {Arot }}$ works equally well from the non-rotating limit up to very rapidly and differentially rotating configurations.

However, a computation of neutron star eigenfrequencies has revealed large errors of up to $50 \%$ for the frequencies of the $F$-mode and its harmonics, which implies that even in spherical symmetry some of the dynamic properties are not captured correctly by any of the effective relativistic potentials investigated. These shortcomings in accurately approximating the correct pulsation frequencies reveals an inherent flaw of the effective relativistic potential approach.

Our findings have important implications for the range of applicability of an effective relativistic potential in an otherwise Newtonian code. The rotation rate of our most rapidly rotating models of (proto-)neutron stars exceed values of $T /|W| \approx 0.3$, which is the expected extreme upper limit for rotational supernova core collapse (Ott et al. 2007b; Dimmelmeier et al. 2008) or accretion-induced collapse (Dessart et al. 2006), and lies well beyond the Kepler limit for uniformly rotating neutron stars ${ }^{5}$. While the potentials $\Phi_{\mathrm{R}}$ and $\Phi_{\mathrm{A}}$, which lack rotational corrections, are already quite unreliable in this regime, such high rotation rates pose no problem for the new potential $\Phi_{\text {Arot }}$. Thus, our new approach is clearly the preferred choice for modeling rapidly rotating compact objects in the framework of pseudoNewtonian simulations.

Acknowledgements. This research has been supported by the DFG (SFB/Transregio 7 and SFB 375) and by the DAAD and IKY (IKYDA German-Greek research travel grant). H.D. is a Marie Curie Intra-European Fellow within the 6th European Community Framework Programme (IEF 040464). It is a pleasure to thank C. D. Ott, A. Marek, and H.-T. Janka for their contributions related to the improved microphysics. We also thank the referee for his suggestions to improve our manuscript.

\footnotetext{
5 Such rotation rates are also above the classical thresholds for the growth of triaxial instabilities on a secular time scale $(T /|W| \geq 0.14)$ and on a dynamical time scale $(T /|W| \geq 0.27)$.
}

\section{References}

Banyuls, F., Font, J. A., Ibáñez, J. M., Martí, J. M., \& Miralles, J. A. 1997, ApJ, 476, 221

Blanchet, L., Damour, T., \& Schäfer, G. 1990, MNRAS, 242, 289

Burrows, A., Livne, E., Dessart, L., Ott, C. D., \& Murphy, J. 2006, ApJ, 640, 878

Burrows, A., Livne, E., Dessart, L., Ott, C. D., \& Murphy, J. 2007, ApJ, 655, 416

Cantiello, M., Yoon, S.-C., Langer, N., \& Livio, M. 2007, A\&A, 465, L29

Centrella, J. M., New, K. C. B., Lowe, L. L., \& Brown, J. D. 2001, ApJ, 550, L193

Cerdá-Durán, P., Faye, G., Dimmelmeier, H., et al. 2005, A\&A, 439, 1033

Dessart, L., Burrows, A., Ott, C. D., et al. 2006, ApJ, 644, 1063

Dessart, L., Burrows, A., Livne, E., \& Ott, C. D. 2007a, ApJ, 669, 585

Dessart, L., Burrows, A., Livne, E., \& Ott, C. D. 2007b, ApJ, 673, L43

Dimmelmeier, H., Font, J. A., \& Müller, E. 2002a, A\&A, 388, 917

Dimmelmeier, H., Font, J. A., \& Müller, E. 2002b, A\&A, 393, 523

Dimmelmeier, H., Novak, J., Font, J. A., Ibáñez, J. M., \& Müller, E. 2005, Phys. Rev. D, 71, 064023

Dimmelmeier, H., Cerdá-Durán, P., Marek, A., \& Faye, G. 2006, in Albert Einstein Century International Conference, ed. J.-M. Alimi, \& A. Füzfa (Melville, USA: American Institute of Physics), AIP Conf. Ser., 861,600

Dimmelmeier, H., Ott, C. D., Janka, H.-T., Marek, A., \& Müller, E. 2007, Phys. Rev. Lett., 98, 251101

Dimmelmeier, H., Ott, C. D., Marek, A., \& Janka, H.-T. 2008, in preparation Finn, L. S. 1989, in Frontiers in Numerical Relativity, ed. C. R. Evans, L. S. Finn, \& D. W. Hobill (Cambridge, UK: Cambridge University Press), 126

Fryer, C. L., \& Heger, A. 2005, ApJ, 623, 302

Heger, A., Langer, N., \& Woosley, S. E. 2000, ApJ, 528, 368

Heger, A., Woosley, S. E., \& Spruit, H. C. 2005, ApJ, 626, 350

Isenberg, J. A. 1978, University of Maryland Preprint, [arXiv:gr-qc/0702113]

Janka, H.-T., Zwerger, T., \& Mönchmeyer, R. 1993, A\&A, 268, 360

Komatsu, H., Eriguchi, Y., \& Hachisu, I. 1989, MNRAS, 237, 355

Langanke, K., \& Martínez-Pinedo, G. 2000, Nucl. Phys. A, 673, 481

Liebendörfer, M. 2005, ApJ, 633, 1042

Liebendörfer, M., Rampp, M., Janka, H.-T., \& Mezzacappa, A. 2005, ApJ, 620, 840

Lin, L.-M., Cheng, K. S., Chu, M.-C., \& Suen, W.-M. 2006, ApJ, 639, 382

MacFadyen, A. I., \& Woosley, S. E. 1999, ApJ, 524, 262

Marek, A., Janka, H.-T., Buras, R., Liebendörfer, M., \& Rampp, M. 2005, A\&A, 443, 201

Marek, A., Dimmelmeier, H., Janka, H.-T., Müller, E., \& Buras, R. 2006, A\&A, 445,273

Mönchmeyer, R., Schäfer, G., Müller, E., \& Kates, R. E. 1991, A\&A, 246, 417

Obergaulinger, M., Aloy, M. A., Dimmelmeier, H., \& Müller, E. 2006, A\&A, 457, 209

Ott, C. D., Ou, S., Tohline, J. E., \& Burrows, A. 2005, ApJ, 625, L119

Ott, C. D., Dimmelmeier, H., Marek, A., et al. 2007a, Phys. Rev. Lett., 98, 261101

Ott, C. D., Dimmelmeier, H., Marek, A., et al. 2007b, Class. Quantum Grav., 24, 139

Ou, S., \& Tohline, J. E. 2006, ApJ, 651, 1068

Ou, S., Tohline, J. E., \& Lindblom, L. 2004, ApJ, 617, 490

Rampp, M., \& Janka, H.-T. 2002, A\&A, 396, 361

Shen, H., Toki, H., Oyamatsu, K., \& Sumiyoshi, K. 1998, Prog. Theor. Phys., 100,1013

Shibata, M., \& Sekiguchi, Y.-I. 2004, Phys. Rev. D, 69, 084024

Shibata, M., \& Sekiguchi, Y.-I. 2005, Phys. Rev. D, 71, 024014

Shibata, M., Karino, S., \& Eriguchi, Y. 2002, MNRAS, 334, L27

Shibata, M., Karino, S., \& Eriguchi, Y. 2003, MNRAS, 343, 619

Straumann, N. 2004, General Relativity with Applications to Astrophysics (Berlin, Germany: Springer)

van Riper, K. A. 1979, ApJ, 232, 558

Weinberg, N. N., \& Quataert, E. 2008, MNRAS, accepted,

[arXiv:0802.1522v2]

Wilson, J. R., Mathews, G. J., \& Marronetti, P. 1996, Phys. Rev. D, 54, 1317

Woosley, S. E., \& Heger, A. 2006, ApJ, 637, 914

Woosley, S. E., Heger, A., \& Weaver, T. A. 2002, Rev. Mod. Phys., 74, 1015

Yoon, S.-C., \& Langer, N. 2005, A\&A, 435, 967

Zwerger, T., \& Müller, E. 1997, A\&A, 320, 209 